\title{
Somatotipo femenino en relación a las marcas en las pruebas de lanzamiento
}

\section{Feminine somato type in relation to the marks in the launching tests}

\author{
Cecilia Alicia Abensur Pinasco*
}

http://dx.doi.org/10.21503/CienciayDesarrollo.2007.v8.05

\section{RESUMEN}

El universo de estudio utilizado en el presente trabajo estuvo conformado por los deportistas atletas a nivel nacional pertenecientes a las ligas atléticas de las diferentes regiones del Perú y que participaron en el Campeonato Nacional de Mayores y Juveniles durante los meses de abril, mayo junio y julio del 2007, en la Villa Deportiva Nacional (VIDENA).

Las muestras se tomaron en horas de la mañana, según el cronograma oficial de la FPA, con el apoyo de los alumnos estudiantes de la Escuela Profesional de Ciencias del Deporte de la U.A.P., bajo la supervisión del licenciado Polo Mayorca Cardich (nivel III ISAK), el magíster Hernando Díaz y la autora. La muestra está representada por 40 atletas damas, y se procedió utilizando el muestreo estratificado.

Se utilizó el criterio de inclusión solamente con atletas mujeres. Como criterio de exclusión se tomó el de no incluir atletas varones ni atletas menores de 14 años.

Se buscó que la correlación entre dos variables reflejara el grado en que las puntuaciones están asociadas. Nos servimos también de la formulación clásica conocida como correlación producto, utilizando Pearson y T de Student.

Las técnicas, instrumentos y fuentes de recolección de datos utilizados en esta investigación corresponden al diseño descriptivo-comparativo, correlativo.

La hipótesis planteada parte de considerar modelos ideales de somatotipo, tomando como referencia a deportistas de nivel mundial y olímpico. En estos modelos predomina el componente mesomórfico sobre el componente endomórfico, y por ende el ectomórfico, lo cual corrobora una serie de estudios de carácter fisiológico y biomecánico, según los cuales se afirma y se comprueba que la potencia y la relación entre peso y talla generan las acciones motrices para la impulsión de la bala.

Los datos obtenidos en el campeonato nacional 2007 describen una población totalmente ajena a esta realidad, pues se ha verificado la predominancia absoluta de la endomorfia sobre la mesomorfia y ectomorfia, considerando que el componente de endomorfia se refiere a la adiposidad relativa del atleta, es decir, representa indirectamente la masa adiposa, no esencial, y que no tiene otro fin fisiológico que el de asegurar una reserva energética para la supervivencia. Por ello, al no ser el componente endomórfico fuente de generación directa de las acciones motrices para actividades que requieren impulsión y potencia, como son las pruebas de lanzamiento, no existiría reciprocidad entre el somatotipo de las lanzadoras nacionales y sus respectivas marcas por especialidad. Los cuadros de correlación entre los componentes del somatotipo y las marcas respectivas por especialidad dan cuenta de esta posibilidad.

Palabras clave: somatotipo, mesomórfico, endomórfico, ectomórfico.

* Directora de la Escuela de Ciencias del Deporte de la Universidad Alas Peruanas 


\section{ABSTRACT}

The Universe of study used of the present work was conformed by all the sport athletes at level National, pertaining to Ligas Athletic of the different regions from Peru. Participants in the National Championship of greater and youthful in the months of April, May June, July of the 2007 in Villa Deportiva Nacional (VIDENA).

The samples were taken in hours the morning according to the official chronogram of the FPA, with the support of the students of the Professional School of Sciences of sports under the Direction and supervision of the Lic. Polo Mayorca Cardich level III ISAK, Mg. Lic. Hernando Díaz and the author del work Lic. Cecilia Alicia Abensur Pinasco. The sample is represented by 40 athletes ladies using the Stratified sampling.

I am only used the criterion of inclusion with athletes women, origin of the athlete. And in the criterion of Exclusion. Volume in consideration of not including to the athletes men, as well as to athletes of 14 years. Looking for the correlation between two variables it reflected the degree in which the scores are associate. The classic, well-known formulation like correlation product, using Pearson and T of Student the Techniques, Instruments and Sources of Data collection Used in this investigation corresponds to the descriptive-comparative, correlative design. The raised hypothesis part to consider obtained through the obtaining of data of sportsmen of world-wide level and Olympic ideal models of somatotipo, in these models predominates the Mesomorfico component on the Endomorfico component therefore of the ectomorfico which corroborates a series of physiological and biomechanics studies of character in where it affirms and verifies that the power, as the relation between weight and carves, generates the motor actions for the impulsion of the bullet

The data collected in national championship 2007 describe a population totally other people's to this reality, predominacía absolute is in a high value of endomorfia on mesomorfia and ectomorfia, considering that the component of endomorfia talks about to the relative adiposity del athlete, is to say indirectly represents nonessential the fatty mass that does not have another physiological aim that the one to assure a power reserve for the survival, by as much, al not to be source of direct generation of the motor actions for activities which they require impulsion and power as they are the launching tests, it would not exist reciprocity between somatotipo of the national throwers and its respective marks by specialty. The pictures of correlation between the components of somatotipo and the respective marks by specialty give account of this possibility.

\section{INTRODUCCIÓN}

Hemos cumplido con nuestro propósito de determinar la relación que existe entre las marcas de las pruebas de lanzamiento y el somatotipo femenino de las atletas lanzadoras de nuestro país. Esto nos ha permitido determinar las características de somatotipo y las diferencias entre unas y otras, al mismo tiempo que nos ha llevado a crear un sistema eficiente de detección y desarrollo de talentos deportivos, así como la ubicación de los atletas en las pruebas correctas. Hemos tenido también la oportunidad de precisar los perfiles de somatotipo en base a los resultados de esta investigación, a fin de afrontar de manera objetiva y responsable los objetivos de los entrenadores. Con todo, es imprescindible la realización de más estudios de esta índole porque nos brindarán información actualizada de las características morfológicas y de la realidad en que nos encontramos, permitiendo así la creación de un sistema eficiente de detección y desarrollo de talentos.

\section{MATERIAL Y MÉTODO}

\section{Formulación del problema de investigación}

La razón de ser de la presente investigación consiste en el desconocimiento de la relación entre el somatotipo de las atletas especializadas en las pruebas de lanzamientos y sus marcas deportivas. Esto nos lleva a formular el problema mediante esta pregunta: ¿cuál es la relación entre el somatotipo de las atletas especializadas en las pruebas de lanzamientos y las marcas deportivas que registran? 


\section{Problema secundario}

A) ¿Cuál es el somatotipo de las deportistas participantes en el Campeonato Nacional 2007 en las pruebas de impulsión de bala?

B) ¿Cuál es el somatotipo de las deportistas participantes en el Campeonato Nacional 2007 en las pruebas de lanzamiento de disco?

C) ¿Cuál es el somatotipo de las deportistas participantes en el Campeonato Nacional 2007 en las pruebas de lanzamiento de martillo?

D) ¿Cuál es el somatotipo de las deportistas participantes en el Campeonato Nacional 2007 en las pruebas de lanzamiento de jabalina?

\section{Delimitación de la investigación}

\section{Delimitación espacial}

La investigación se realizará en la Villa Deportiva Nacional (VIDENA), institución que cuenta con autorización del IPD para ejercer funciones de desarrollo de eventos deportivos atléticos a nivel de Lima metropolitana y campeonatos nacionales e internacionales, según el calendario de actividades 2007 . En su recinto alberga a los atletas hombres y mujeres de todas las regiones del Perú participantes en tales eventos.

\section{Delimitación temporal}

El diseño es prospectivo, y se desarrollará en el Campeonato Nacional de Mayores, los días 17,18 y 19 del mes de mayo y los días 1,2 y 3 del mes de junio del año 2007, así como en el Campeonato Metropolitano, Campeonato Nacional de Juveniles del año 2007.

\section{Delimitación cuantitativa}

La cantidad de atletas participantes en el Campeonato Nacional de Mayores y juveniles es de 300 atletas, de los cuales un $10 \%$ son lanzadores, constituyéndose estos últimos en nuestra población y muestra (véase Cap. IV). La generalización de los resultados será en el total de atletas lanzadoras mayores y juveniles, afiliadas a la FPA y participantes en los campeonatos indicados.

\section{Objetivos de la investigación}

\section{Objetivo general}

Determinar la relación existente entre el somatotipo de las atletas de alto nivel especializadas en pruebas de lanzamientos y la marca deportiva que registran en la(s) prueba(s) de su especialidad

\section{Objetivos especificos}

a. Identificar el somatotipo de las deportistas participantes en el Campeonato Nacional de Mayores y Juveniles del año 2007 en las pruebas de lanzamiento de disco.

b. Identificar el somatotipo de las deportistas participantes en el Campeonato Nacional de Mayores y Juveniles del año 2007 en las pruebas de la impulsión de bala.

c. Identificar el somatotipo de las deportistas participantes en el Campeonato Nacional de Mayores y Juveniles del año 2007 en las pruebas de lanzamiento de martillo.

d. Identificar el somatotipo de las deportistas participantes en el Campeonato Nacional de Mayores y Juveniles del año 2007 en las pruebas de lanzamiento, de jabalina.

\section{Hipotesis y variables}

\section{Hipótesis general}

Existe relación entre el somatotipo que presentan las atletas de alto nivel especializadas en pruebas de lanzamientos y la marca deportiva que registran en la(s) prueba(s) de su especialidad. Esta relación se caracteriza por la predominancia de uno de los componentes del somatotipo, en dependencia de la modalidad de lanzamiento.

\section{Hipótesis especificas}

a. Existe relación entre uno de los componentes del somatotipo y la marca obtenida en la prueba de impulsión de la bala. 
b. Existe relación entre uno de los componentes del somatotipo y la marca obtenida en la prueba de lanzamiento del disco.

c. Existe relación entre uno de los componentes del somatotipo y la marca obtenida en la prueba de lanzamiento de jabalina.

d. Existe relación entre uno de los componente del somatotipo y la marca obtenida en la prueba de lanzamiento de martillo.

\section{Variables e indicadores (de la hipótesis generaly} especifica)

Variable independiente " $\mathrm{X}$ " - Indicadores Somatotipo
a. Mesomorfo
b. Endomorfo
c. Ectomorfo

Variables dependientes "Y" - Indicadores

Marca atlética registrada

a. Resultados obtenidos en el Campeonato Nacional 2007 en la impulsión de bala.

b. Resultados obtenidos en el Campeonato Nacional 2007 en los lanzamientos de disco.

c. Resultados obtenidos en el campeonato Nacional 2007 en los lanzamientos de jabalina.

d. Resultados obtenidos en el Campeonato Nacional 2007 en los lanzamientos de martillo

Variable interviniente

Lugar de procedencia: Lima, Ica, la Libertad, Tacna, Pucallpa.

Operacionalización de variables: Variables - Definición - Dimensión - Indicadores - Escala

\section{Operacionalización de variables}

\begin{tabular}{|c|c|c|c|c|}
\hline VARIABLE & DEFINICIÓN & DIMENSIÓN & INDICADORES & ESCALA \\
\hline $\begin{array}{l}\text { Variable } \\
\text { independiente: } \\
\text { somatotipo }\end{array}$ & $\begin{array}{l}\text { Es una técnica para describir y } \\
\text { analizar las variaciones de la figura } \\
\text { humana. Su nomenclatura se basa } \\
\text { en las tres capas y se determina } \\
\text { por la evaluación integral de los } \\
\text { tres componentes: endomórfico, } \\
\text { mesomórfico y ectomórfico. } \\
\text { "El somatotipo permite una } \\
\text { clasificación de la forma en escalas } \\
\text { que puedan ser expresadas con un } \\
\text { simple valor numérico" } 1(1)\end{array}$ & $\begin{array}{l}\text { Atletas mujeres del } \\
\text { Campeonato Nacional de } \\
\text { Mayores y juveniles, año } \\
2007 .\end{array}$ & $\begin{array}{l}\text { Ectomorfo } \\
\text { Endomorfo } \\
\text { Mesomorfo y } \\
13 \text { categorías }\end{array}$ & Ordinal \\
\hline
\end{tabular}

\begin{tabular}{|c|c|c|c|c|}
\hline VARIABLE & DEFINICIÓN & DIMENSIÓN & INDICADORES & $\begin{array}{l}\text { ESCALA DE } \\
\text { MEDICIÓN }\end{array}$ \\
\hline $\begin{array}{l}\text { Variable } \\
\text { dependiente: } \\
\text { marcas registradas } \\
\text { oficialmente de las } \\
\text { atletas }\end{array}$ & $\begin{array}{l}\text { Son las marcas registradas } \\
\text { oficialmente en los } \\
\text { campeonatos nacionales de } \\
\text { mayores y juveniles del año } \\
2007 \text {. }\end{array}$ & $\begin{array}{l}\text { Competidoras del } \\
\text { Campeonato Nacional de } \\
\text { Mayores y Juveniles, año } \\
2007 .\end{array}$ & $\begin{array}{l}\text { - Marcas registradas en la } \\
\text { impulsión de bala. } \\
\text { - Marcas registradas en el } \\
\text { lanzamiento de disco. } \\
\text { - Marcas registradas en el } \\
\text { lanzamiento de jabalina. } \\
\text { - Marcas registradas en el } \\
\text { lanzamiento de martillo. }\end{array}$ & $\begin{array}{l}\text { Proporcional o de } \\
\text { razón. }\end{array}$ \\
\hline $\begin{array}{l}\text { Variable } \\
\text { interviniente: } \\
\text { región de } \\
\text { procedencia. }\end{array}$ & $\begin{array}{l}\text { Llamamos región geográfica } \\
\text { de procedencia a aquella en la } \\
\text { cual el atleta ha desarrollado } \\
\text { sus dos últimos macrociclos de } \\
\text { entrenamiento. }\end{array}$ & $\begin{array}{l}\text { Regiones geográficas } \\
\text { costa, sierra y selva. }\end{array}$ & Circunstancial & Nominal \\
\hline
\end{tabular}

(1) http://www.patriciaminuchin.com.ar/Publicado/06Cineantropometria.htm 


\section{Operacionalización de las hipótesis específicas}

\begin{tabular}{|c|c|c|c|}
\hline HIPÓTESIS & $\begin{array}{c}\text { VARIABLE } \\
\text { INDEPENDIENTE }\end{array}$ & $\begin{array}{c}\text { VARIABLE } \\
\text { DEPENDIENTE }\end{array}$ & $\begin{array}{c}\text { VARIABLE } \\
\text { INTERVINIENTE }\end{array}$ \\
\hline $\begin{array}{l}\text { He } 1 \\
\text { Existe relación entre la predominancia } \\
\text { del componente endomórfico y la } \\
\text { marca obtenida en la prueba de la } \\
\text { impulsión de bala. }\end{array}$ & $\begin{array}{l}\text { - Mesomorfo } \\
\text { - Endomorfo } \\
\text { - } \text { Ectomorfo } \\
\text { y sus trece componentes. }\end{array}$ & $\begin{array}{l}\text { Todos los resultados } \\
\text { oficiales de los } \\
\text { campeonatos nacionales } \\
\text { de atletismo, año } 2007 \text {. }\end{array}$ & $\begin{array}{l}\text { Las regiones de } \\
\text { procedencia: costa, sierra } \\
\text { y selva. }\end{array}$ \\
\hline $\begin{array}{l}\text { He } 2 \\
\text { Existe relación directa entre la } \\
\text { predominancia de la categoría del } \\
\text { mesoectomórfico y la marca obtenida } \\
\text { en la prueba de lanzamiento del } \\
\text { disco. }\end{array}$ & $\begin{array}{l}\text { - Mesomorfo } \\
\text { - Endomorfo } \\
\text { - } \text { Ectomorfo } \\
\text { y sus trece componentes. }\end{array}$ & $\begin{array}{l}\text { Todos los resultados } \\
\text { oficiales de los } \\
\text { campeonatos nacionales } \\
\text { de atletismo, año } 2007 \text {. }\end{array}$ & $\begin{array}{l}\text { - Las regiones de } \\
\text { procedencia: costa, sierra } \\
\text { y selva. }\end{array}$ \\
\hline $\begin{array}{l}\text { He } 3 \\
\text { Existe relación directa entre la } \\
\text { predominancia de la categoría del } \\
\text { mesoectomórfico y la marca obtenida } \\
\text { en la prueba de lanzamiento de } \\
\text { jabalina. }\end{array}$ & $\begin{array}{l}\text { - Mesomorfo } \\
\text { - Endomorfo } \\
\text { - Ectomorfo } \\
\text { y sus trece componentes. }\end{array}$ & $\begin{array}{l}\text { Todos los resultados } \\
\text { oficiales de los } \\
\text { campeonatos nacionales } \\
\text { de atletismo, año } 2007 \text {. }\end{array}$ & $\begin{array}{l}\text { - Las regiones de } \\
\text { procedencia: costa, sierra } \\
\text { y selva. }\end{array}$ \\
\hline $\begin{array}{l}\text { He } 4 \\
\text { Existe relación directa entre la } \\
\text { predominancia de la categoría del } \\
\text { mesoectomórfico y la marca obtenida } \\
\text { en la prueba de lanzamiento de } \\
\text { martillo. }\end{array}$ & $\begin{array}{l}\text { - Mesomorfo } \\
\text { - Endomorfo } \\
\text { - Ectomorfo } \\
\text { y sus trece componentes. }\end{array}$ & $\begin{array}{l}\text { Todos los resultados } \\
\text { oficiales de los } \\
\text { campeonatos nacionales } \\
\text { de atletismo, año } 2007 \text {. }\end{array}$ & $\begin{array}{l}\text { - Las regiones de } \\
\text { procedencia: costa, sierra } \\
\text { y selva. }\end{array}$ \\
\hline
\end{tabular}

Tipos y niveles de investigación

\section{(a) Niveles}

Nivel aplicativo.

\section{(b) Tipos}

\section{Cuantitativa positivista \\ Comprende la interpretativa y estadística}

\section{Diseño de la investigación}

La investigación proyectada corresponde al diseño descriptivo-comparativo, pues tiene por finalidad describir e interpretar sistemáticamente las principales modalidades de formación o de cambio de un fenómeno para avanzar en la solución de los problemas que se presentan (Van Dalen y Meyer, 1971; Briones, 1986); procurando determinar las características del fenómeno en una determinada circunstancia témporo-espacial. a. Tipo, niveles de investigación y diseño descriptivo comparativo complejo

Diagrama:

$$
\begin{array}{llllllll}
\mathrm{M}_{1} & \mathrm{O}_{1} & & & & & \\
& & & \sim & & \\
\mathrm{M}_{2} & \mathrm{O}_{2} & \mathrm{O}_{1} & = & \mathrm{O}_{2} & = & \mathrm{O}_{3} & = \\
& & & \neq & & & \\
\mathrm{M}_{3} & \mathrm{O}_{2} & & & & & & \\
\mathrm{M}_{4} & \mathrm{O}_{4} & & & & &
\end{array}
$$

\section{Significado de los símbolos:}

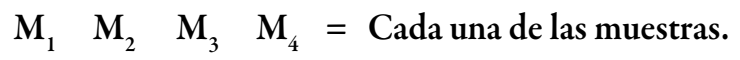

$\begin{array}{llll}\mathrm{O}_{1} & \mathrm{O}_{2} & \mathrm{O}_{3} & \mathrm{O}_{4}\end{array}=$ Información de cada muestra.

$=; \neq ; \sim \sim \quad$ = Igual, diferente, semejante.

Donde $\mathrm{M}_{1}, \mathrm{M}_{2}, \mathrm{M}_{3}$ y $\mathrm{M}_{4}$ representan a cada una de las muestras; $\mathrm{O}_{1}, \mathrm{O}_{2}, 0_{3}, \mathrm{O}_{4}$, la información (observaciones) recolectada en cada una de dichas muestras; los $\mathrm{O}_{1}$ a $\mathrm{O}_{\mathrm{x}}$ en la parte lateral del diagrama nos indican las comparaciones que se llevan a cabo entre cada una de las muestras, pudiendo estas observaciones, resultados o información ser: iguales $(=)$, diferentes $(\not)$ o semejantes $(\sim)$ con respecto a la otra. 
b. Correlacional

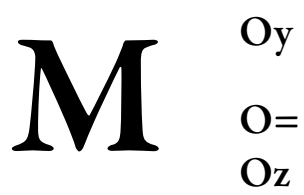

Donde:

$\mathrm{M} \quad=$ Muestra

$\mathrm{y}, \mathrm{x}, \mathrm{z},=$ Subíndices. Observaciones obtenidas de cada una de las tres variables.

$\mathrm{R}=$ Indica la posible relación entre las variables estudiadas.

\section{Universo - Población}

\section{(a) Universo}

El universo de estudio del presente trabajo lo conforman todas las deportistas atletas a nivel nacional. Ellas pertenecen a las ligas atléticas de las diferentes regiones del Perú participantes en el Campeonato Nacional de Mayores y Juveniles del 2007 en los meses de abril, mayo junio, julio del 2007 en la VILLA DEPORTIVA NACIONAL (VIDENA) de la Federación Peruana de Atletismo (F.P.A).

\section{(b) Población}

Atletas participantes en le Campeonato Nacional de Mayores y Juveniles del año 2007 en la sede de la VIDENA de la F.P.A.

\section{Muestra}

La muestra está representada por 30 atletas damas, donde $\mathbf{N}=\mathbf{n}$.

\section{(a) Tamaño de la muestra}

(b) Muestreo: se utilizará el muestreo estratificado.

(c) Criterio de inclusión

Atletas (mujeres) participantes en las pruebas de lanzamiento del Campeonato Nacional de Mayores y Juveniles del año 2007. Teniendo en cuenta que en los campeonatos nacionales de juveniles y mayores también compiten las atletas de la categoría menores, las mediciones se realizarán desde los 15 años a más. Así mismo se tendrá como referencia la procedencia de las atletas, así como si son casadas o solteras.

\section{Criterio de exclusión}

No se incluirán a los atletas varones.

No se incluirán a atletas menores de 14 años.

\begin{tabular}{|c|c|c|c|c|}
\hline Prueba & COSTA & SELVA & SIERRA & totales \\
\hline DISCO & 9 & 1 & 0 & 10 \\
\hline BALA & 7 & 1 & 2 & 10 \\
\hline MARTILLO & 9 & 0 & 0 & 9 \\
\hline JABALINA & 5 & 0 & 0 & 5 \\
\hline Totales: & 30 & 2 & 2 & 34 \\
\hline
\end{tabular}

Técnicas, instrumentos y fuentes de recolección de datos

Técnicas

Se utilizarán los distintos instrumentos para la obtención de información referida a las medidas antropométricas, dando las características morfológicas y de composición corporal que se detallan en los cuadros de la página siguiente.

\section{Instrumentos}

Cintas antropométricas: para los perímetros.

Talla: distancia máxima entre la región plantar y el vértex.

Estadiómetro fijo: graduado en centímetros y medias unidades.

Estadiómetro fijo: instrumento utilizado para medir la estatura y la altura sentado.

Peso: una de las variables antropométricas más comunes.

Balanzas de plataforma con pesas deslizables, electrónicas. 


\begin{tabular}{l|l|l}
$\begin{array}{c}\text { VARIABLE } \\
\text { INDEPENDIENTE }\end{array}$ & \multicolumn{1}{c}{ INDICADORES } & \multicolumn{1}{c}{ TÉCNICAS } \\
\hline & $\begin{array}{l}\text { Ectomorfo, mesomorfo, endomorfo y sus } 13 \\
\text { componentes: }\end{array}$ & $\begin{array}{l}\text { Toma de mediciones } \\
\text { antropométricas } \\
\text { validada por ISAK. } \\
\text { (International Society }\end{array}$ \\
1. & Endoectomórfico. & for the Advancement of \\
2. & Endomorfo balanceado & Kinanthropometry) \\
3. & Endomesomórfico & \\
4. & Endomorfo-Mesomorfo & \\
5. & Mesendomórfico. & \\
6. & Mesomorfo balanceado & \\
7. & Mesoectomórfico. & \\
8. & Ectomorfo - Mesomorfo. & \\
9. & Ectomesomórfico & \\
10. Ectomorfo balanceado & \\
11. Ectoendomórfico & \\
12. Ectomorfo-Endomorfo & \\
13. & Central & \\
\hline
\end{tabular}

\begin{tabular}{c|l|l}
\multicolumn{1}{c|}{\begin{tabular}{c}
\multicolumn{1}{c|}{ VARIABLE } \\
DEPENDIENTE
\end{tabular}} & \multicolumn{1}{|c}{ INDICADORES } & \multicolumn{1}{c}{ TÉCNICAS } \\
\hline Marcas registradas & $\begin{array}{l}\text { Marcas oficiales registradas en el } \\
\text { Campeonato Nacional y Juvenil del año } \\
2007\end{array}$ & $\begin{array}{l}\text { Recolección de datos } \\
\text { y fichas de resultados } \\
\text { oficiales, y por página } \\
\text { web de la F.P.A. }\end{array}$ \\
\hline
\end{tabular}

\begin{tabular}{c|c|c}
\hline VARIABLE & INDICADORES & TÉCNICAS \\
INTERVINIENTE & Regiones físicas costa, sierra y selva. & Información textual \\
\hline $\begin{array}{c}\text { Regiones } \\
\text { geográficas }\end{array}$ & & \\
\hline
\end{tabular}

\section{Calibres para la medición de pliegues cutá-} neos: se utilizarán los calibres Slim Guide.

Antropómetros: confeccionados sobre la base de aluminio para la medición de diámetros pequeños y grandes.

Antropómetros de diferentes longitudes: que van de los $20,0 \mathrm{~cm}$ a los $60,0 \mathrm{~cm}$. Escalas divididas en centímetros y milímetros.
Segmómetro: fabricado a partir de una cinta de acero de carpintero.

Calibres de ramas curvas: utilizados para la medición del diámetro anteroposterior del tórax, con dos ramas o brazos curvos.

Caja antropométrica: que debe presentar dimensiones con longitudes de todos los lados, de aproximadamente $40 \mathrm{CNT}$. 


\section{FICHA DE CINEANTROPOMETRÍA INTERNACIONAL DE MEDICIONES ANTROPOMÉTRICAS}

\section{CINEANTROPOMETRÍA INTERNACIONAL - MEDICIONES ANTROPOMÉTRICAS}

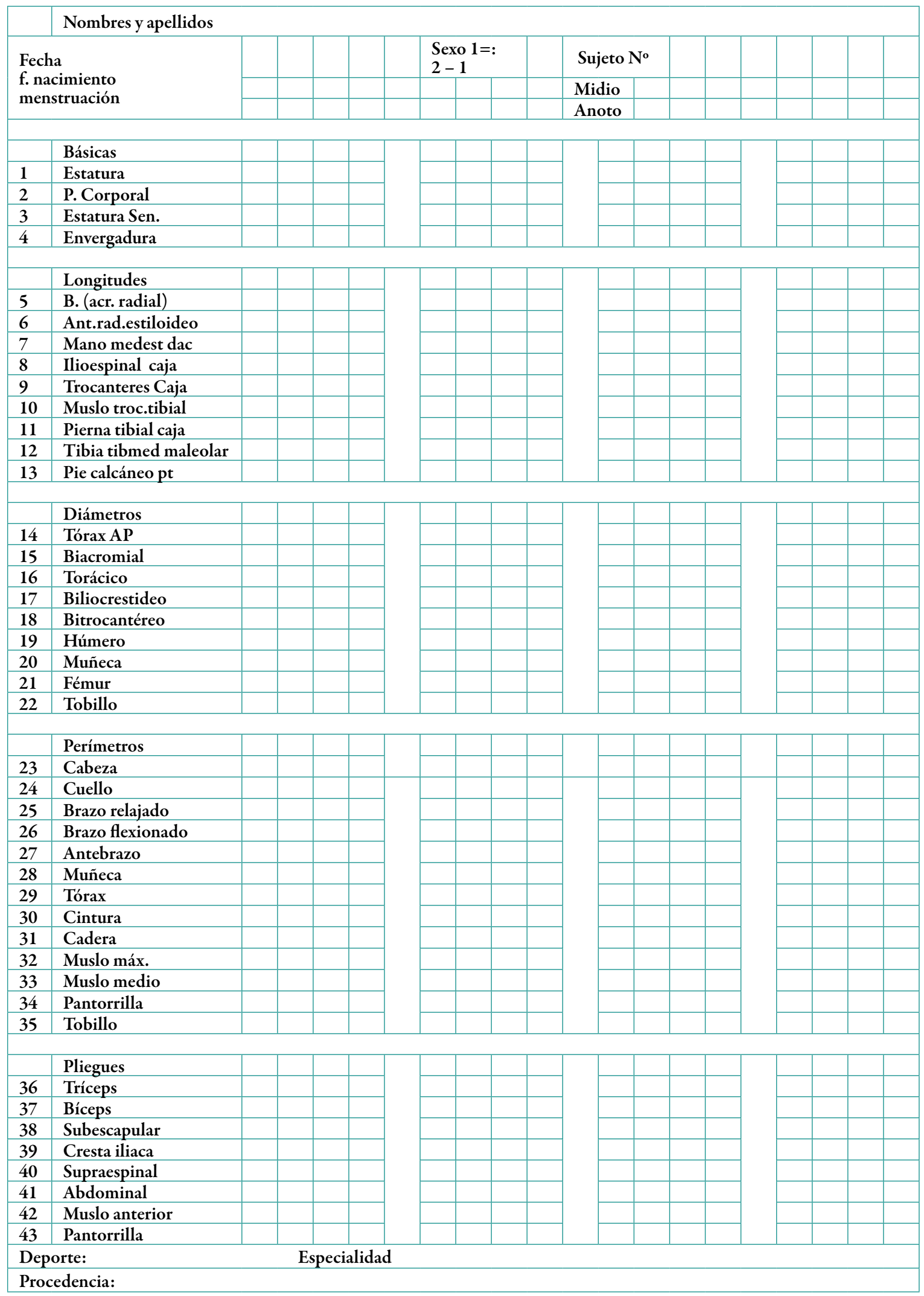




\section{Técnicas de Procesamiento y Análisis de la Información}

Selección de Técnicas de acuerdo a las variables - indicadores

\begin{tabular}{|c|c|c|}
\hline $\begin{array}{c}\text { VARIABLE } \\
\text { INDEPENDIENTE }\end{array}$ & \multicolumn{2}{|c|}{ TÉCNICAS } \\
\hline SOMATOTIPO & \multicolumn{2}{|c|}{$\begin{array}{l}\text { Mediciones antropométricas, con kid antropométrico } \\
\text { de ISAK (International Society for the Advancement of } \\
\text { Kinanthropometry }\end{array}$} \\
\hline $\begin{array}{c}\text { VARIABLE } \\
\text { DEPENDIENTE }\end{array}$ & \multicolumn{2}{|c|}{ TÉCNICAS } \\
\hline MARCAS REGISTRADAS & \multicolumn{2}{|c|}{$\begin{array}{l}\text { Registro de las marcas del Campeonato Nacional de Mayores y } \\
\text { Juveniles, año } 2007 .\end{array}$} \\
\hline $\begin{array}{c}\text { VARIABLE } \\
\text { INTERVINIENTE }\end{array}$ & \multicolumn{2}{|c|}{ TÉCNICAS } \\
\hline $\begin{array}{l}\text { REGIONES FÍSICAS } \\
\text { GEOGRÁFICAS }\end{array}$ & \multicolumn{2}{|l|}{ Referencias textuales. } \\
\hline \multicolumn{3}{|c|}{ Matriz y tripartitas de datos } \\
\hline UNIVERSO & POBLACIÓN & MUESTRA \\
\hline Atletas de todo el Perú & $\begin{array}{l}\text { Atletas mujeres participantes } \\
\text { en el Campeonato Nacional de } \\
\text { Mayores y Juveniles, año } 2007\end{array}$ & $\mathrm{n}=34$, donde $\mathrm{N}=\mathrm{n}$ \\
\hline
\end{tabular}

\section{Utilización de procesador sistematizador computarizado}

Para el procesamiento de datos se utilizara el software estadístico SPSS 13, MINITAB y la hoja de cálculo Excel. Y para la evaluación de datos antropométricos, el software especial para los resultados presentado por ISAK (International Asociation for kinanthropometry)

\section{Pruebas estadísticas - contrastación}

\section{Elr de Pearson}

La correlación entre dos variables refleja el grado en que las puntuaciones están asociadas. La formulación clásica es conocida como correlación producto. La formula suele aparecer expresada como:

$$
r=\frac{\sigma_{X Y}}{\sigma_{X} \cdot \sigma_{Y}}
$$

\begin{tabular}{c|l}
$\begin{array}{c}\text { Valor del } \\
\text { coeficiente de Pearson }\end{array}$ & \multicolumn{1}{c}{$\begin{array}{c}\text { Grado de correlación } \\
\text { entre las variables }\end{array}$} \\
\hline$r=0$ & Ninguna correlación \\
\hline$r=1$ & Correlación positiva perfecta \\
\hline $0<r<1$ & Correlación positiva \\
\hline$r=-1$ & Correlación negativa perfecta \\
\hline$-1<r<0$ & Correlación negativa \\
\hline
\end{tabular}

Contraste sobre la diferencia de medias -T Student: diferencia de medias poblacionales ${ }^{\mu_{1}-\mu_{2}}$

Muestras independientes

Si puede suponerse que las varianzas de ambas poblaciones son iguales, el intervalo de 
confianza para la diferencia de medias poblacionales está centrado en la diferencia de las medias muestrales, siendo sus límites superior e inferior:

$$
\left(\bar{X}_{1}-\bar{X}_{2}\right) \pm t_{\alpha / 2} S \sqrt{\frac{1}{n_{3}}+\frac{1}{n_{2}}}
$$

$t^{\alpha} / 2$ es el valor crítico correspondiente al grado de confianza $1-^{\alpha}$ de la distribución $t$ de Student con $n 1+n 2-2$ grados de libertad, $y$

$$
S=\sqrt{\frac{\left(n_{1}-1\right) S_{1}^{2}+\left(n_{2}-1\right) S_{2}^{2}}{n_{1}+n_{2}-2}}
$$

es una estimación de la desviación típica común a ambas poblaciones, obtenida a partir de las varianzas de las dos muestras. En la práctica, si $\mathrm{n}_{1} \mathrm{y}_{2}$ son moderadamente grandes, el valor crítico $t^{\alpha} / 2$ se aproxima, como ya se ha visto anteriormente, a los valores de la distribución normal.

Si las varianzas poblacionales no pueden suponerse iguales, los límites del intervalo de confianza son:

$$
\left(\bar{X}_{1}-\bar{X}_{2}\right) \pm t_{\alpha / 2} \sqrt{\frac{S_{P}}{n_{s}}+\frac{S_{l}}{n_{2}}}
$$

El valor crítico $t^{\alpha} / 2$ corresponde a una distribución $t$ cuyos grados de libertad se calculan en base a ambos tamaños muestrales y a las desviaciones típicas de cada grupo, según la corrección propuesta por Dixon y Massey:

$$
g \cdot 1=\frac{\left(\frac{s_{i}^{2}}{m_{1}}+\frac{s_{2}}{n_{2}}\right)^{2}}{\left(\frac{s_{1}^{2}}{n_{1}}\right)^{2}\left(\frac{1}{n+1}\right)+\left(\frac{s_{2}^{2}}{m_{3}}\right)^{2}\left(\frac{1}{n+1}\right)}-2
$$

\section{Contrastación y comprobación de hipótesis}

Proceso de contrastación de las hipótesis - estadísticas

\section{Matriz tripartita de datos}

\begin{tabular}{l|ll}
\multicolumn{1}{c|}{ Universo } & \multicolumn{1}{c}{ Población } & \multicolumn{1}{c}{ Muestra } \\
\cline { 1 - 1 } $\begin{array}{l}\text { Atletas especialistas en la prueba de } \\
\text { impulsión de bala. }\end{array}$ & \multicolumn{1}{c}{5 atletas } \\
\cline { 1 - 1 } $\begin{array}{l}\text { Atletas especialistas en la prueba de } \\
\text { lanzamiento de disco. }\end{array}$ & $\begin{array}{l}\text { Atletas mujeres participantes en el } \\
\text { Campeonato Nacional de Juveniles }\end{array}$ & 15 atletas \\
\cline { 1 - 1 } $\begin{array}{l}\text { Atletas especialistas en la prueba de } \\
\text { lanzamiento de jabalina. }\end{array}$ & & 9 atletas \\
\cline { 1 - 1 } $\begin{array}{l}\text { Atletas especialistas en la prueba de } \\
\text { lanzamiento del martillo. }\end{array}$ & & 5 atletas
\end{tabular}

Contraste entre variable independiente con la dependiente

Se realizó la correlación entre las variables independientes, que son los tres componentes del somatotipo, y la variable dependiente con las marcas logradas en los campeonatos nacionales de juveniles y mayores del año 2007, por especialidad de lanzamiento. 

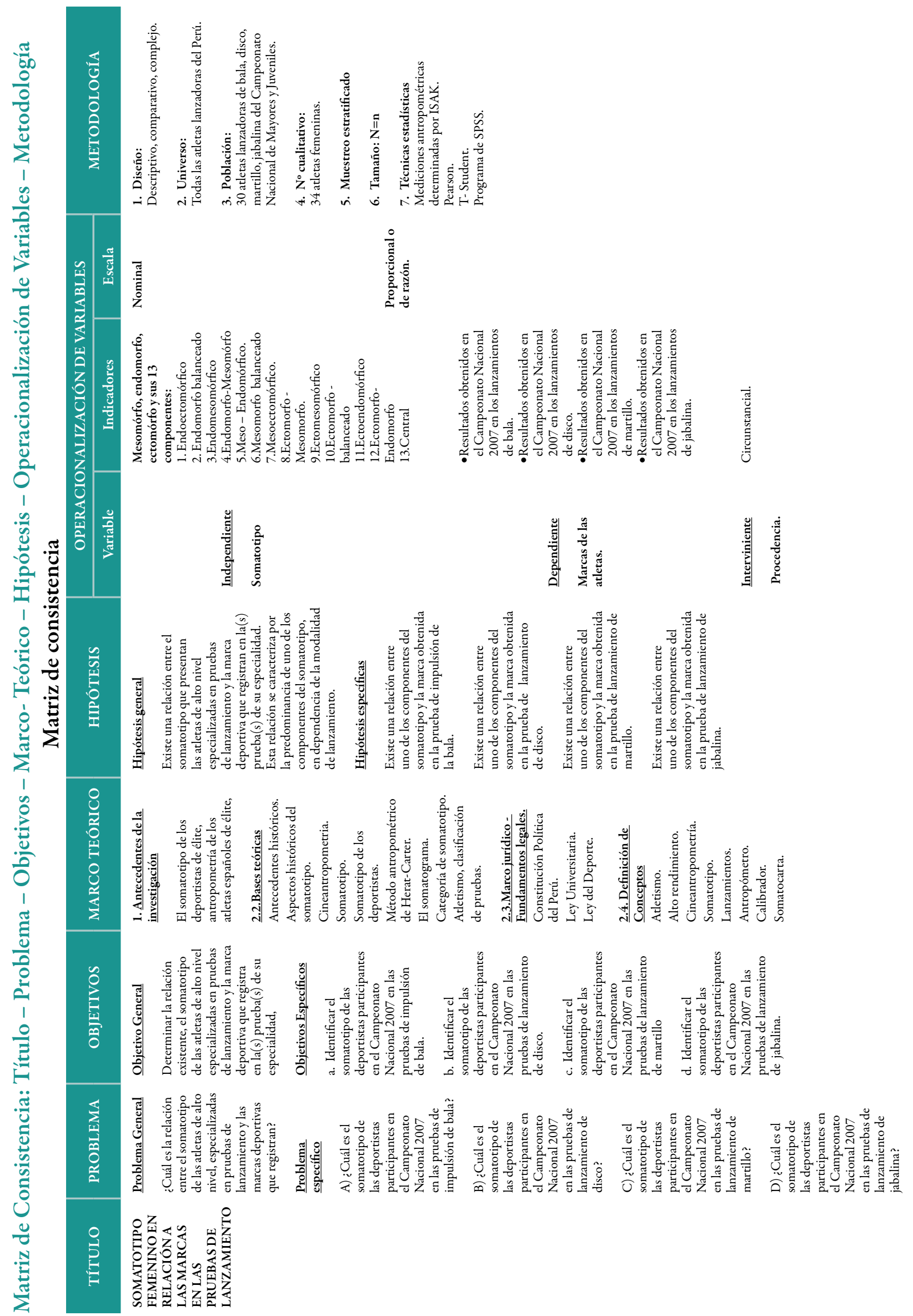
Análisis de los datos recolectados

\begin{tabular}{|c|c|c|c|c|c|c|c|c|c|c|c|c|c|}
\hline COD & APELLIDOS Y NOMBRES & SEXO & $\mathrm{Xc}$ & Endo & Meso & ecto & $\mathrm{X}$ & $\mathrm{Y}$ & ESPECIALIDAD & MARCA & DDS & Decision & Predominancia \\
\hline 71 & PARODI CHALE, ESTELA & $\mathrm{F}$ & 68,70 & 6,49 & 5,19 & 0,32 & $-6,17$ & 3,56 & MARTILLO & 43,5 & 13,26 & Diferencia Significativa & Endomorfo - Mesomórfico \\
\hline 73 & PEREDA DAMIANI, ANA LUCÍA & $\mathrm{F}$ & 71,09 & 6,66 & 5,97 & 0,38 & $-6,28$ & 4,89 & MARTILLO & 24,19 & 13,41 & Diferencia Significativa & Endomorfo - Mesomórfico \\
\hline 85 & TUSSO QUISPE, DIANA & $\mathrm{F}$ & 73,78 & 6,85 & 6,33 & 0,10 & $-6,75$ & 5,71 & MARTILLO & 33,1 & 14,08 & Diferencia Significativa & Endomorfo - Mesomórfico \\
\hline 92 & CÓRDOVA, KARINA & $\mathrm{F}$ & 87,68 & 7,72 & 5,44 & 0,14 & $-7,58$ & 3,01 & MARTILLO & 42,64 & 15,29 & Diferencia Significativa & Endomorfo - Mesomórfico \\
\hline 93 & ROMERO, EVELYN & $\mathrm{F}$ & 78,14 & 7,14 & 7,51 & 0,10 & $-7,04$ & 7,79 & MARTILLO & 34,55 & 14,49 & Diferencia Significativa & Endomorfo - Mesomorfo \\
\hline 34 & AGURTO MUÑOZ, THALIA MISSIEL & $\mathrm{F}$ & 67,07 & 6,38 & 3,91 & 2,43 & $-3,94$ & $-0,99$ & JABALINA & 23,48 & 10,40 & Diferencia Significativa & Endomorfo - Mesomórfico \\
\hline 47 & FLORES GÓMEZ, DIANA STEPHANIE & $\mathrm{F}$ & 60,89 & 5,91 & 4,65 & 1,44 & $-4,47$ & 1,96 & JABALINA & 34,37 & 11,02 & Diferencia Significativa & Endomorfo - Mesomórfico \\
\hline 61 & JASIMOTO ÁVILA, KAREN & $\mathrm{F}$ & 48,62 & 4,89 & 3,44 & 1,70 & $-3,19$ & 0,29 & JABALINA & 33,53 & 9,60 & Diferencia Significativa & Endomorfo - Mesomórfico \\
\hline 63 & MEDINA CORREA, ROSA & $\mathrm{F}$ & 71,19 & 6,67 & 6,81 & 0,10 & $-6,57$ & 6,85 & JABALINA & 24,22 & 13,82 & Diferencia Significativa & Endomorfo - Mesomorfo \\
\hline 73 & PEREDA DAMIANI, ANA LUCÍA & $\mathrm{F}$ & 71,09 & 6,66 & 5,97 & 0,38 & $-6,28$ & 4,89 & JABALINA & 24,08 & 13,41 & Diferencia Significativa & Endomorfo - Mesomórfico \\
\hline 83 & SERRANO CANALES, MARÍA STEFANY & F & 37,98 & 3,89 & 3,40 & 3,05 & $-0,84$ & $-0,14$ & JABALINA & 22,83 & 7,98 & Diferencia Significativa & Central \\
\hline 89 & MENDOZA, ALEJANDRA & $\mathrm{F}$ & 48,62 & 4,89 & 5,59 & 0,62 & $-4,27$ & 5,67 & JABALINA & 16,7 & 10,78 & Diferencia Significativa & Endomorfo - Mesomorfo \\
\hline 90 & PEREZ, ACELA & $\mathrm{F}$ & 25,25 & 2,53 & 3,55 & 2,22 & $-0,32$ & 2,36 & JABALINA & 25,4 & 7,86 & Diferencia Significativa & Endomorfo - Mesomorfo \\
\hline 91 & PAREDES, NOELIA & $\mathrm{F}$ & 33,98 & 3,48 & 1,39 & 2,56 & $-0,93$ & $-3,25$ & JABALINA & 41,83 & 8,01 & Diferencia Significativa & Endomorfo - Ectomórfico \\
\hline 34 & AGURTO MUÑOZ, THALIA MISSIEL & $\mathrm{F}$ & 67,07 & 6,38 & 3,91 & 2,43 & $-3,94$ & $-0,99$ & DISCO & 20,87 & 10,40 & Diferencia Significativa & Endomorfo - Mesomórfico \\
\hline 36 & ARAUJO ROLDÁN, GABRIELA & $\mathrm{F}$ & 72,42 & 6,76 & 5,04 & 1,02 & $-5,73$ & 2,30 & DISCO & 24,14 & 12,66 & Diferencia Significativa & Endomorfo - Mesomórfico \\
\hline 37 & BORJA MEJÍA, SHADDAI DAMARIS & $\mathrm{F}$ & 60,85 & 5,91 & 4,00 & 1,37 & $-4,54$ & 0,72 & DISCO & 25,59 & 11,11 & Diferencia Significativa & Endomorfo - Mesomórfico \\
\hline 38 & CAMMINATI PÉREZ, MARÍA DEL ROCÍO & $\mathrm{F}$ & 76,38 & 7,02 & 7,54 & 0,10 & $-6,92$ & 7,95 & DISCO & 20,64 & 14,33 & Diferencia Significativa & Endomorfo - Mesomorfo \\
\hline 39 & CARPIO CUADRA, GIULIANA ADELINA & $\mathrm{F}$ & 45,43 & 4,60 & 2,53 & 2,71 & $-1,89$ & $-2,24$ & DISCO & 28,37 & 8,50 & Diferencia Significativa & Endomorfo Balanceado \\
\hline 40 & CARPIO CUADRA, PAOLA ELISA & $\mathrm{F}$ & 80,64 & 7,30 & 5,89 & 0,21 & $-7,09$ & 4,27 & DISCO & 27,03 & 14,57 & Diferencia Significativa & Endomorfo - Mesomórfico \\
\hline 47 & FLORES GÓMEZ, DIANA STEPHANIE & $\mathrm{F}$ & 60,89 & 5,91 & 4,65 & 1,44 & $-4,47$ & 1,96 & DISCO & 27,23 & 11,02 & Diferencia Significativa & Endomorfo - Mesomórfico \\
\hline 50 & GAMBINI PIMINCHUMO, SILVANA & $\mathrm{F}$ & 45,45 & 4,60 & 5,86 & 0,93 & $-3,67$ & 6,18 & DISCO & 31,39 & 10,10 & Diferencia Significativa & Endomorfo - Mesomorfo \\
\hline 64 & MONTEVERDE SUEYRAS, CLAUDIA & $\mathrm{F}$ & 35,50 & 3,64 & 2,50 & 2,30 & $-1,34$ & $-0,94$ & DISCO & 29,23 & 8,18 & Diferencia Significativa & Endomorfo Balanceado \\
\hline 71 & PARODI CHALE, ESTELA & $\mathrm{F}$ & 68,70 & 6,49 & 5,19 & 0,32 & $-6,17$ & 3,56 & DISCO & 30,41 & 13,26 & Diferencia Significativa & Endomorfo - Mesomórfico \\
\hline 73 & PEREDA DAMIANI, ANA LUCÍA & $\mathrm{F}$ & 71,09 & 6,66 & 5,97 & 0,38 & $-6,28$ & 4,89 & DISCO & 33,3 & 13,41 & Diferencia Significativa & Endomorfo - Mesomórfico \\
\hline 84 & SORIANO ALFARO, DALY ALESSANDRA & $\mathrm{F}$ & 46,02 & 4,66 & 5,24 & 1,55 & $-3,11$ & 4,27 & DISCO & 15,68 & 9,52 & Diferencia Significativa & Endomorfo - Mesomorfo \\
\hline 87 & VILLAR PIMENTEL, LEYDY & $\mathrm{F}$ & 68,90 & 6,51 & 5,58 & 0,90 & $-5,61$ & 3,75 & DISCO & 15,98 & 12,49 & Diferencia Significativa & Endomorfo - Mesomórfico \\
\hline 91 & PAREDES, NOELIA & $\mathrm{F}$ & 33,98 & 3,48 & 1,39 & 2,56 & $-0,93$ & $-3,25$ & DISCO & 32,14 & 8,01 & Diferencia Significativa & Endomorfo - Ectomórfico \\
\hline 92 & CÓRDOVA, KARINA & $\mathrm{F}$ & 87,68 & 7,72 & 5,44 & 0,14 & $-7,58$ & 3,01 & DISCO & 27,64 & 15,29 & Diferencia Significativa & Endomorfo - Mesomórfico \\
\hline 39 & CARPIO CUADRA, GIULIANA ADELINA & $\mathrm{F}$ & 45,43 & 4,60 & 2,53 & 2,71 & $-1,89$ & $-2,24$ & BALA & 7,5 & 8,44 & Diferencia Significativa & Endomorfo Balanceado \\
\hline 40 & CARPIO CUADRA, PAOLA ELISA & $\mathrm{F}$ & 80,64 & 7,30 & 5,89 & 0,21 & $-7,09$ & 4,27 & BALA & 8,93 & 14,57 & Diferencia Significativa & Endomorfo - Mesomórfico \\
\hline 64 & MONTEVERDE SUEYRAS, CLAUDIA & $\mathrm{F}$ & 35,50 & 3,64 & 2,50 & 2,30 & $-1,34$ & $-0,94$ & BALA & 10,14 & 8,18 & Diferencia Significativa & Endomorfo Balanceado \\
\hline 71 & PARODI CHALE, ESTELA & $\mathrm{F}$ & 68,70 & 6,49 & 5,19 & 0,32 & $-6,17$ & 3,56 & BALA & 10,99 & 13,26 & Diferencia Significativa & Endomorfo - Mesomórfico \\
\hline 73 & PEREDA DAMIANI, ANA LUCÍA & $\mathrm{F}$ & 71,09 & 6,66 & 5,97 & 0,38 & $-6,28$ & 4,89 & BALA & 8,99 & 13,41 & Diferencia Significativa & Endomorfo - Mesomórfico \\
\hline & LANZAMIENTO - ARGENTINA DAMAS & & & 4,70 & 5,30 & 1,00 & $-3,70$ & 5,60 & & & & & \\
\hline
\end{tabular}

Figura 1. Descripción de cada componente del somatotipo de la especialidad de lanzamiento en el Campeonato Nacional, año 2007.

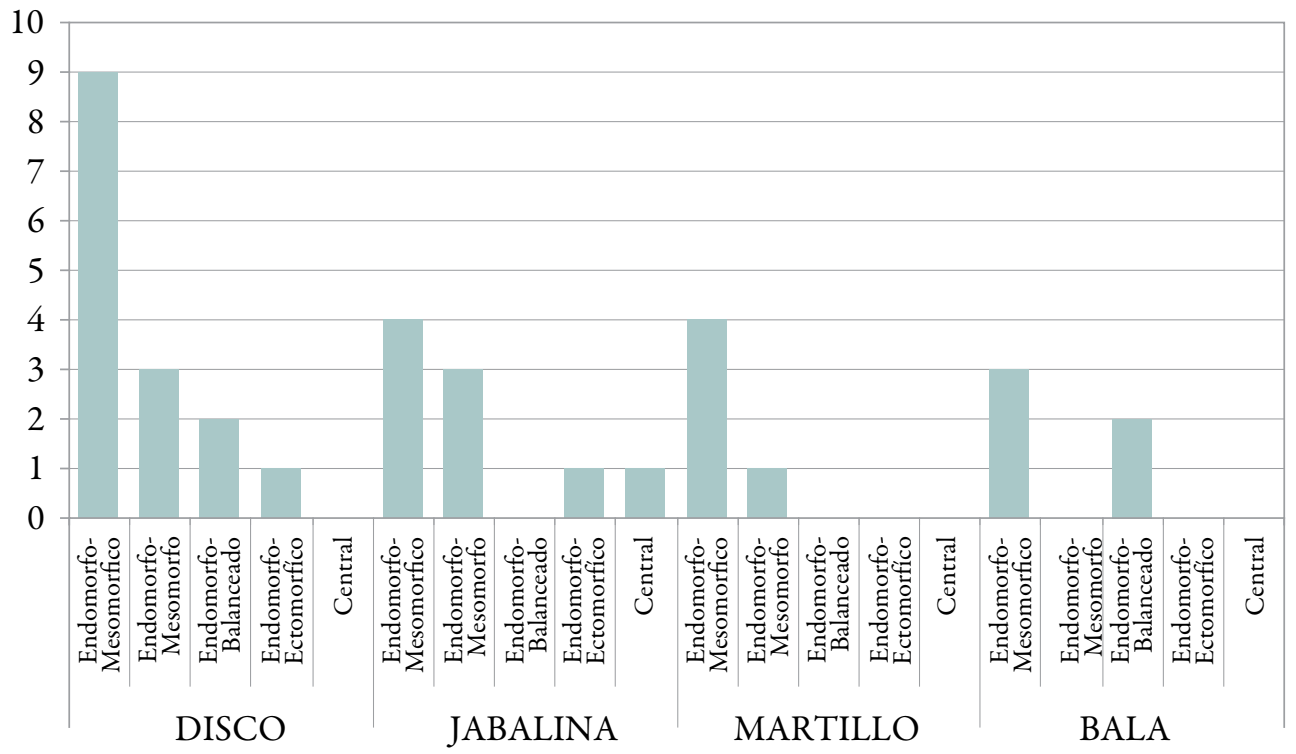

Figura 2. Valor predominante del componente endomórfico sobre el mesomórfico y ectomórfico. 


\section{SOMATOTIPO POR ESPECIALIDADES}
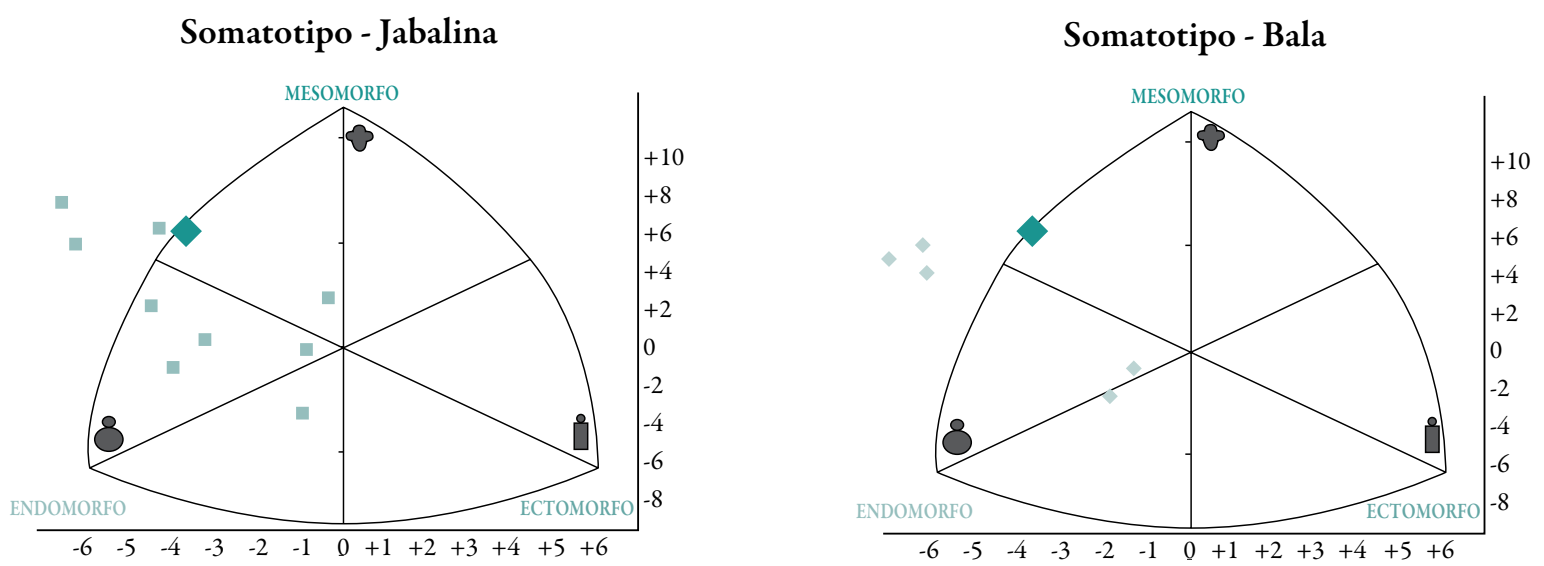

Somatotipo - Martillo
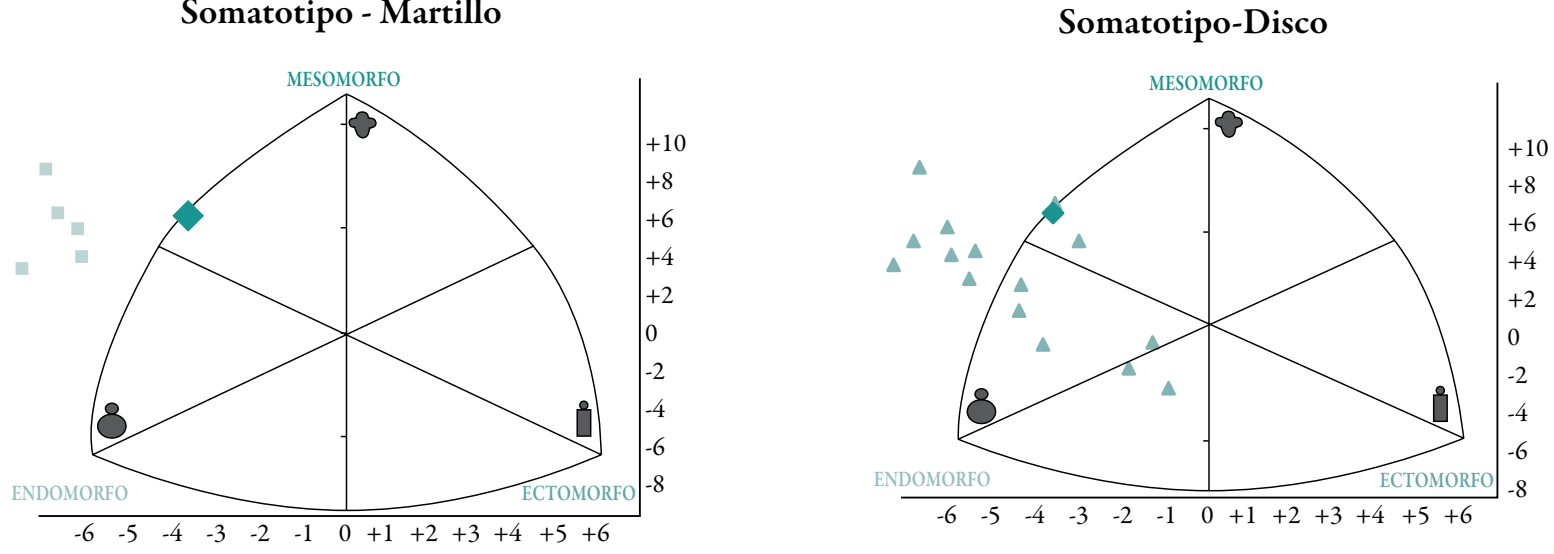

Distribución de frecuencias o histogramas por variables de la información obtenida

Porcentaje de predominancia del somatotipo en los atletas

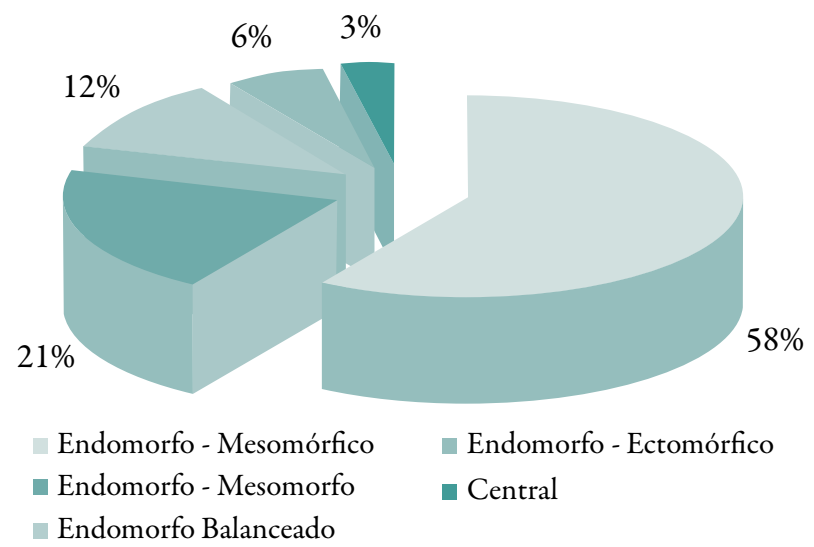

Figura 3. Se observa que en la característica del somatotipo del atleta nacional de impulsión de bala predomina el componente endomórfico.

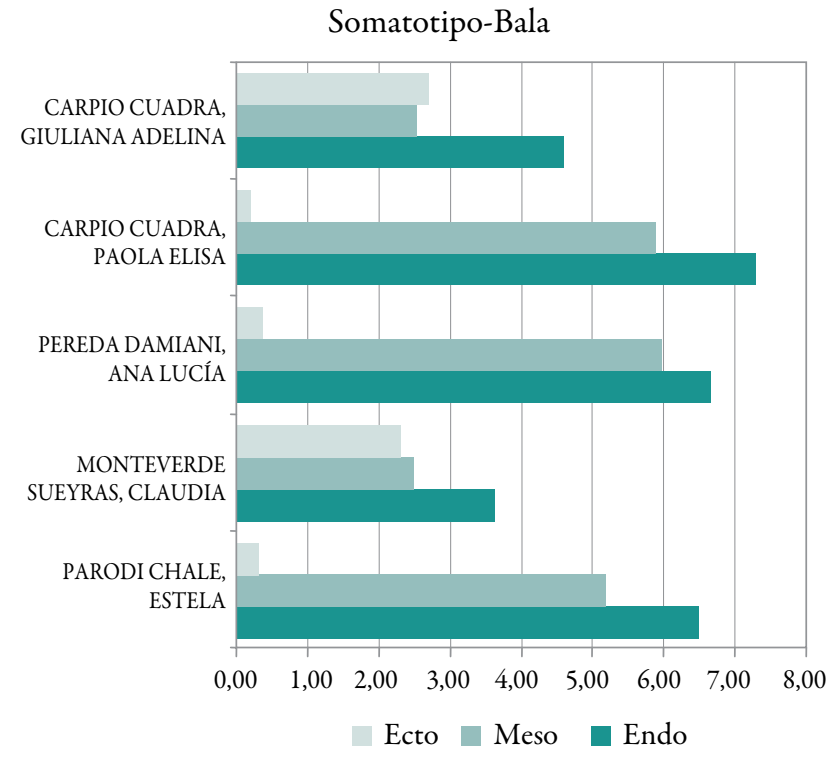

Figura 4. Medida de cada componente del somatotipo de la especialidad de la impulsión de bala. 


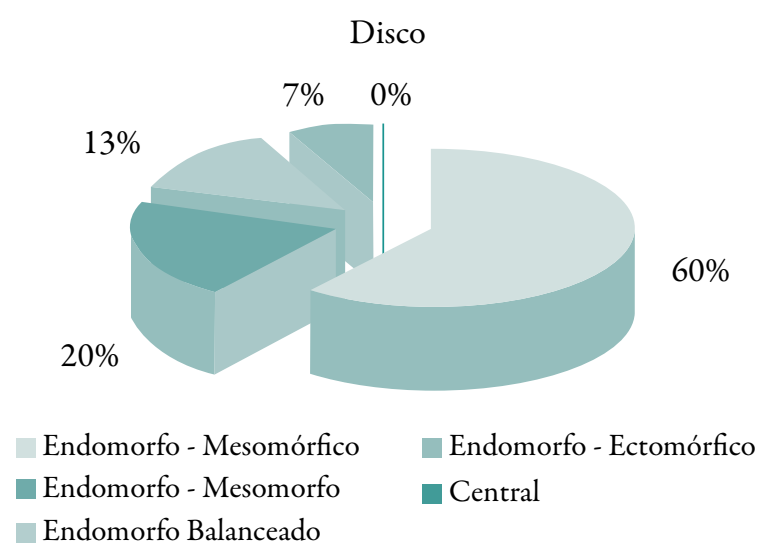

Figura 5. Se observa que en la característica del somatotipo de la atleta nacional de lanzamiento de disco predomina el componente endomórfico.

\section{Somatotipo-Disco}

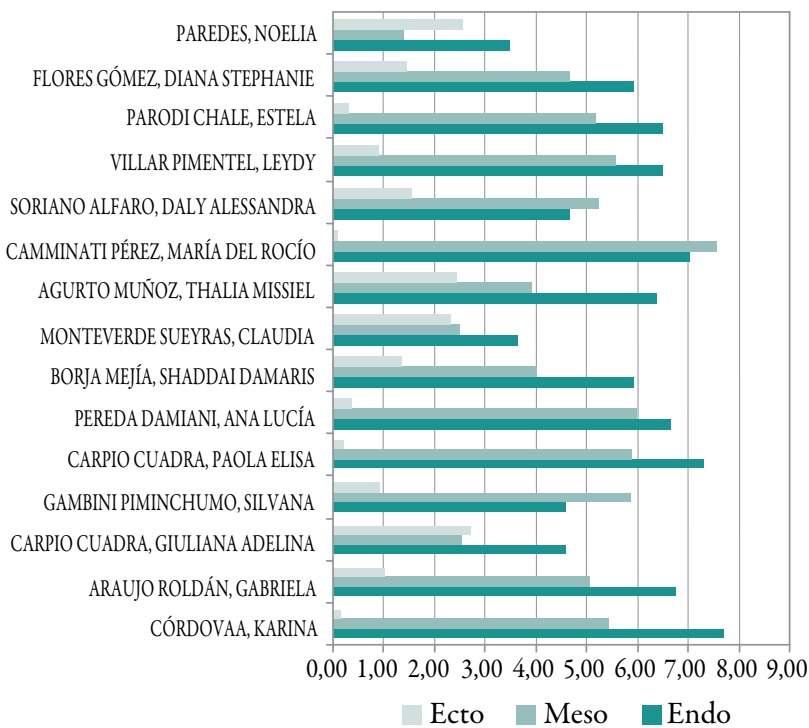

Figura 6. Medida de cada componente del somatotipo de la especialidad de lanzamiento de disco.

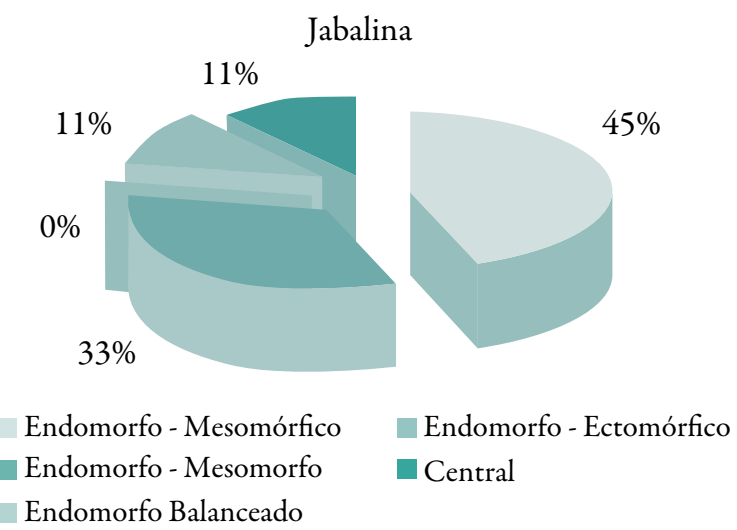

Figura 7. Se observa que en la característica del somatotipo de la atleta nacional de lanzamiento de jabalina predomina el componente endomórfico.

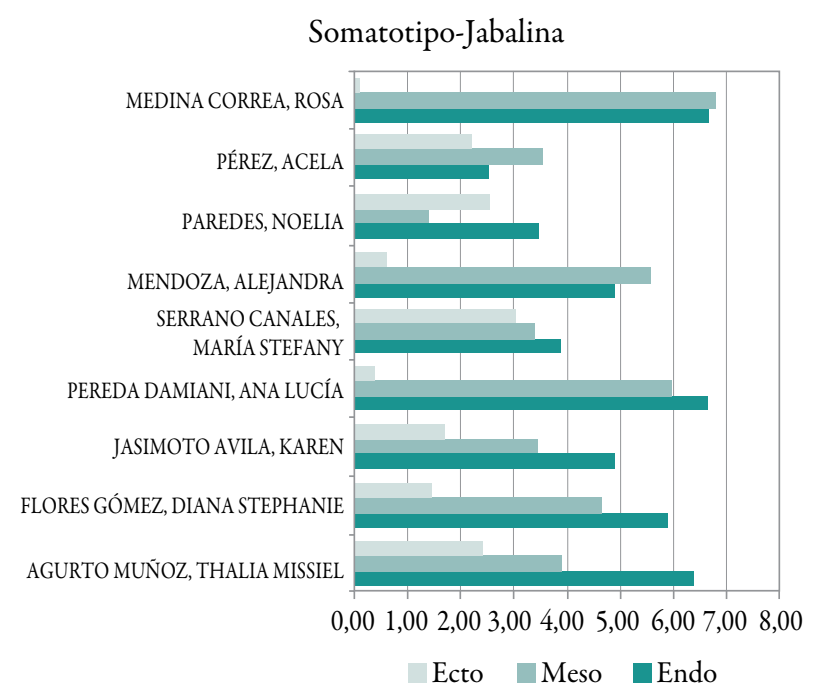

Figura 8. Medida de cada componente del somatotipo de la especialidad de lanzamiento de jabalina.

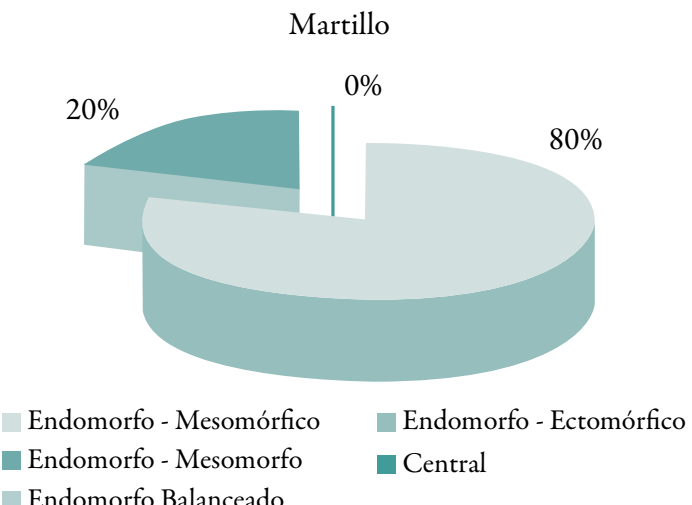

Figura 9. Se observa que en la característica del somatotipo de la atleta nacional de lanzamiento de martillo predomina el componente endomórfico.

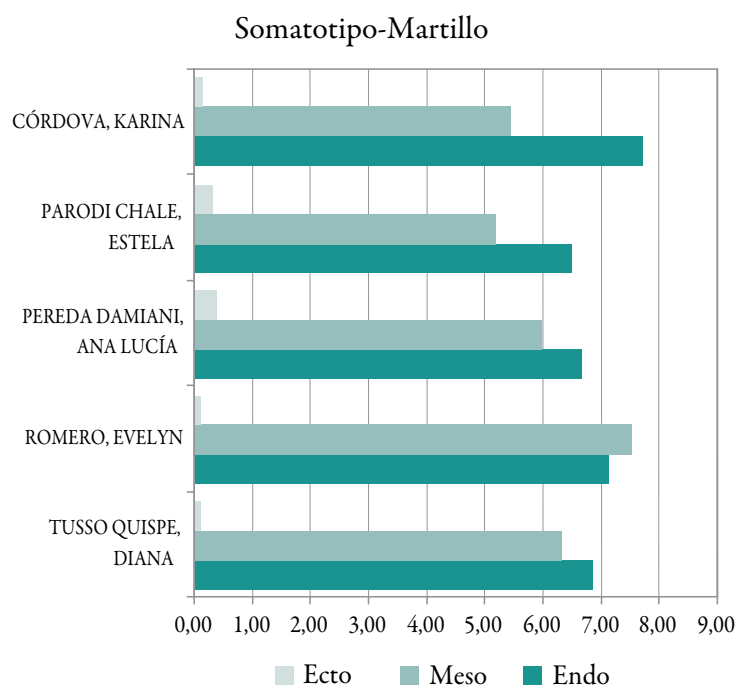

Figura 10. Medida de cada componente del somatotipo de la especialidad de lanzamiento de martillo. 
Dispersión de las marcas obtenidas por especialidades vs. la medida mesomórfica

Las figuras que relacionan el componente mesomórfico con las marcas de bala y disco muestran una ligera tendencia positiva en el caso de esta última; sin embargo, no permite afirmar una reciprocidad entre estos valores debido a las características del somatotipo de las lanzadoras de disco nacional en las que predo- mina el componente de endomorfia. Esto puede deberse a las particularidades del somatotipo de estas atletas, con predominancia del componente endomórfico.

Esta afirmación adquiere mayor validez en el caso de la correlación respectiva del somatotipo de las lanzadoras de jabalina y martillo con sus respectivas marcas, donde no se observa reciprocidad alguna con el componente mesomórfico.
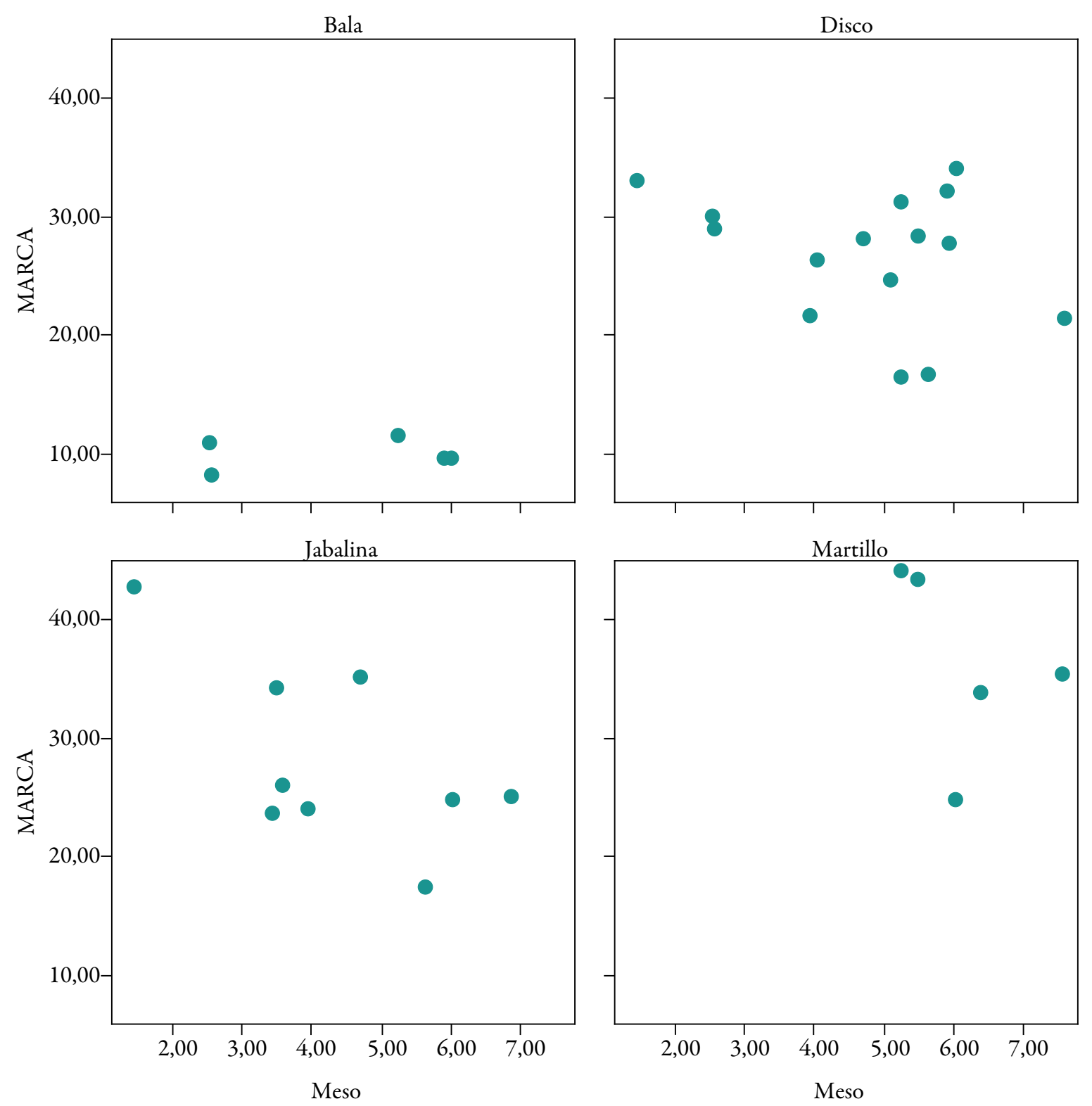
Dispersión de las marcas obtenidas por especialidades vs. la medida endomórfica

Las figuras que relacionan el componente endomórfico con las marcas de bala, disco, jabalina y martillo no muestran asociación alguna, lo que confirma la representatividad como componente del somatotipo de la adi- posidad relativa, cuyo papel en la generación de la potencia del impulso provoca una ligera tendencia positiva en el caso de esta última; sin embargo, no permite afirmar una reciprocidad entre estos valores debido a las características del somatotipo de las lanzadoras de disco nacional en las que predomina el componente endomórfico.
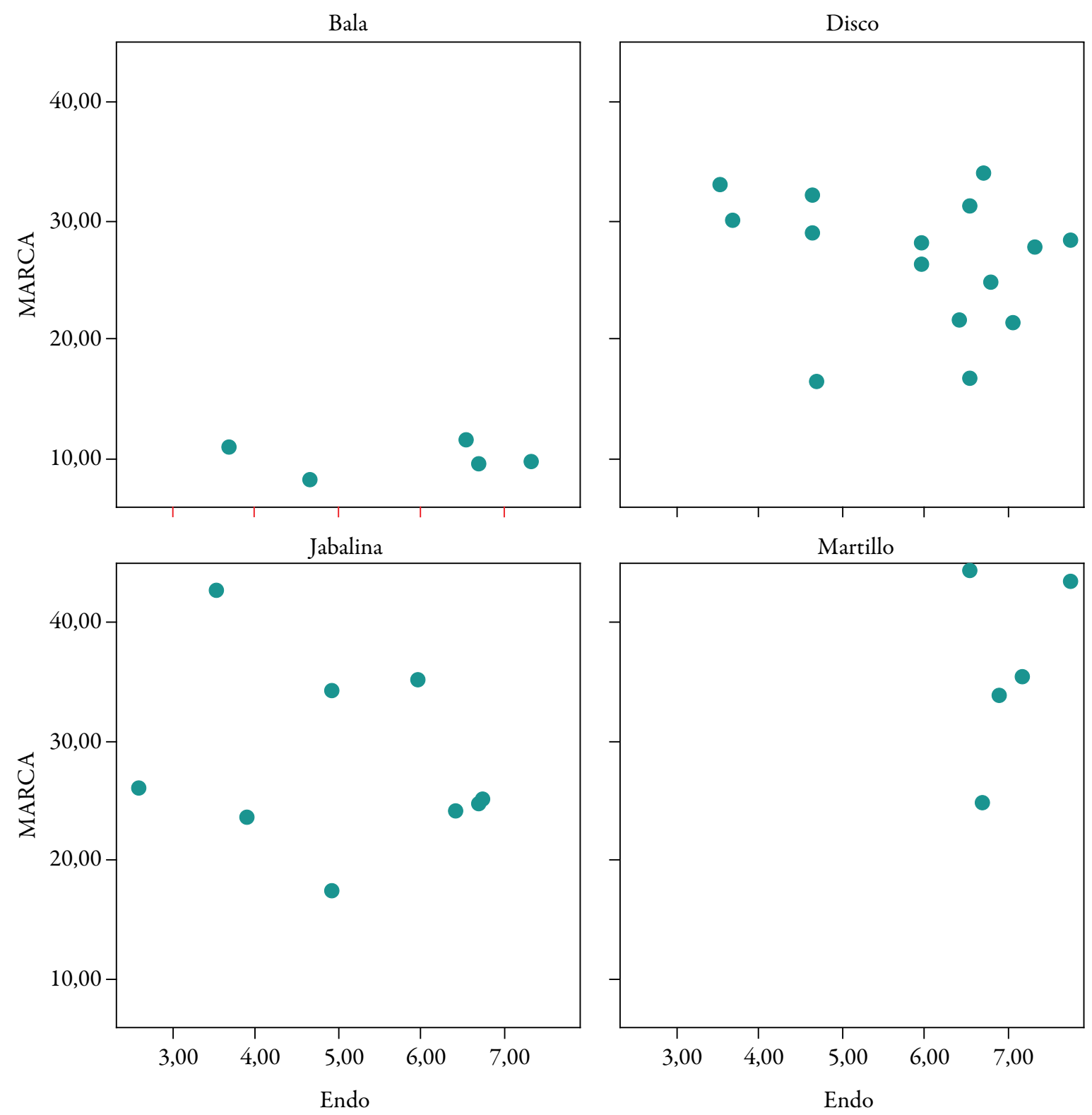
Dispersión de las marcas obtenidas por especialidades vs. la medida ectomórfica

Las figuras que relacionan el componente ectomórfico con las marcas de disco y jabalina muestran una ligera tendencia positiva en la aso- ciación entre la ectomorfia, referida a la distribución de los componentes de la endomorfia, y la mesomorfia sobre los segmentos corporales y las marcas respectivas. Así mismo, al asociar el componente ectomórfico con las marcas de martillo y bala, no se hace evidente una relación.
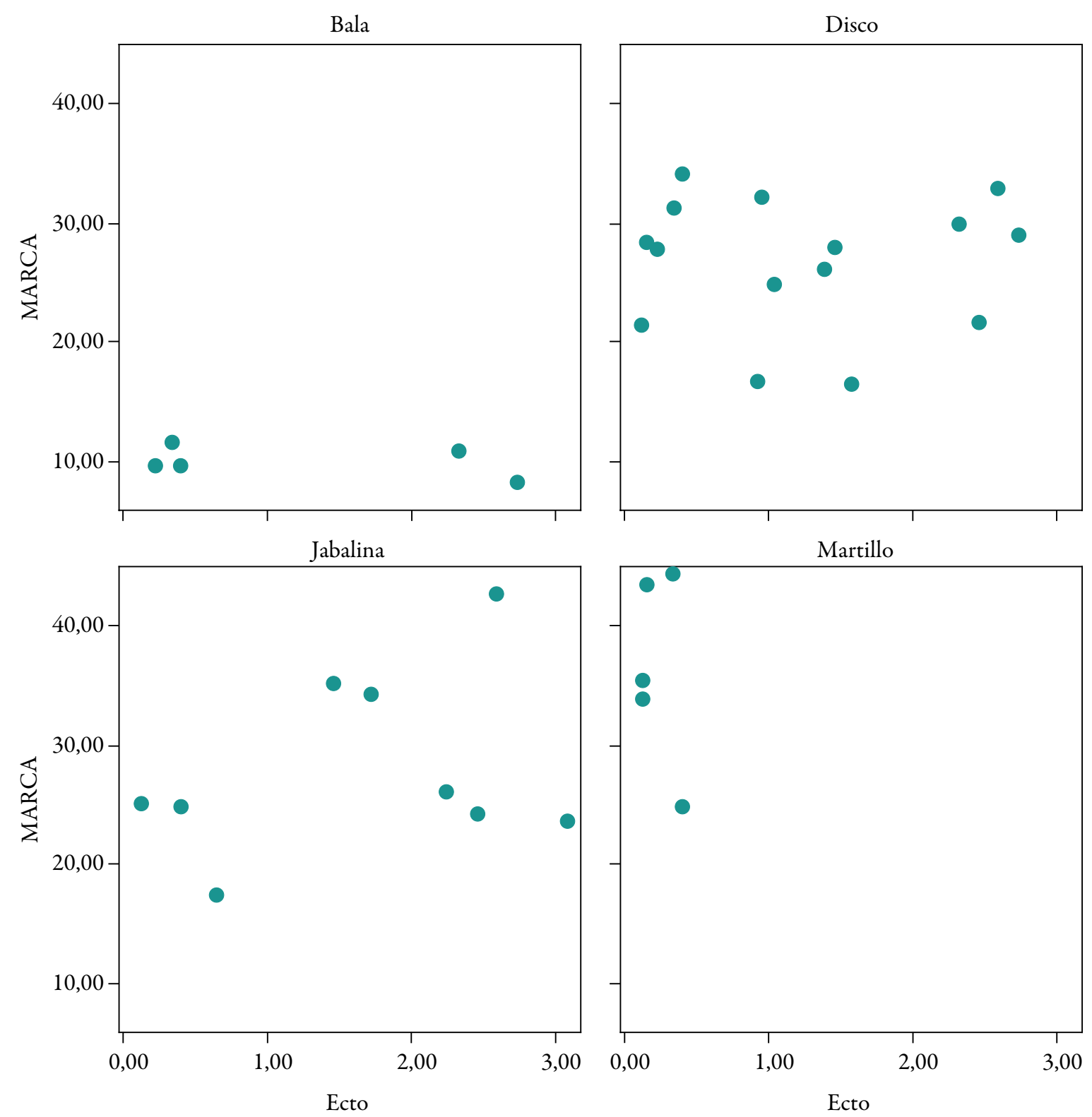
No existe una correlación significativa entre los componentes endo, meso y ecto y las marcas registradas.

Correlations

\begin{tabular}{lllccc}
\multicolumn{2}{c}{ ESPECIALIDAD } & & Endo & Meso & ecto \\
\multirow{2}{*}{ Bala } & \multirow{2}{*}{ MARCA } & Pearson Correlation & 0,085 & 0,220 & $-0,412$ \\
& & Sig. $(2$-tailed $)$ & 0,892 & 0,722 & 0,491 \\
& & N & 5 & 5 & 5 \\
\hline \multirow{2}{*}{ Disco } & \multirow{2}{*}{ MARCA } & Pearson Correlation & $-0,224$ & $-0,310$ & 0,014 \\
& & Sig. $(2$-tailed $)$ & 0,423 & 0,261 & 0,961 \\
& & N & 15 & 15 & 15 \\
\hline \multirow{2}{*}{ Jabalina } & \multirow{2}{*}{ MARCA } & Pearson Correlation & $-0,232$ & $-0,645$ & 0,333 \\
& & Sig. $(2$-tailed $)$ & 0,549 & 0,061 & 0,381 \\
& & $N$ & 9 & 9 & 9 \\
\hline \multirow{2}{*}{ Martillo } & \multirow{2}{*}{ MARCA } & Pearson Correlation & 0,339 & $-0,432$ & $-0,280$ \\
& & Sig. $(2$-tailed $)$ & 0,576 & 0,468 & 0,648 \\
& & $\mathrm{~N}$ & 5 & 5 & 5 \\
\hline
\end{tabular}

Se realizó la prueba de $\mathrm{T}$ de Student para disco, encontrándose que no existe una diferencomparar las poblaciones independientes de las cia significativa en los componentes somatotípiatletas que compiten en la impulsión de bala y cos de ambas especialidades.

\section{Bala y Disco}

Independent Samples Test

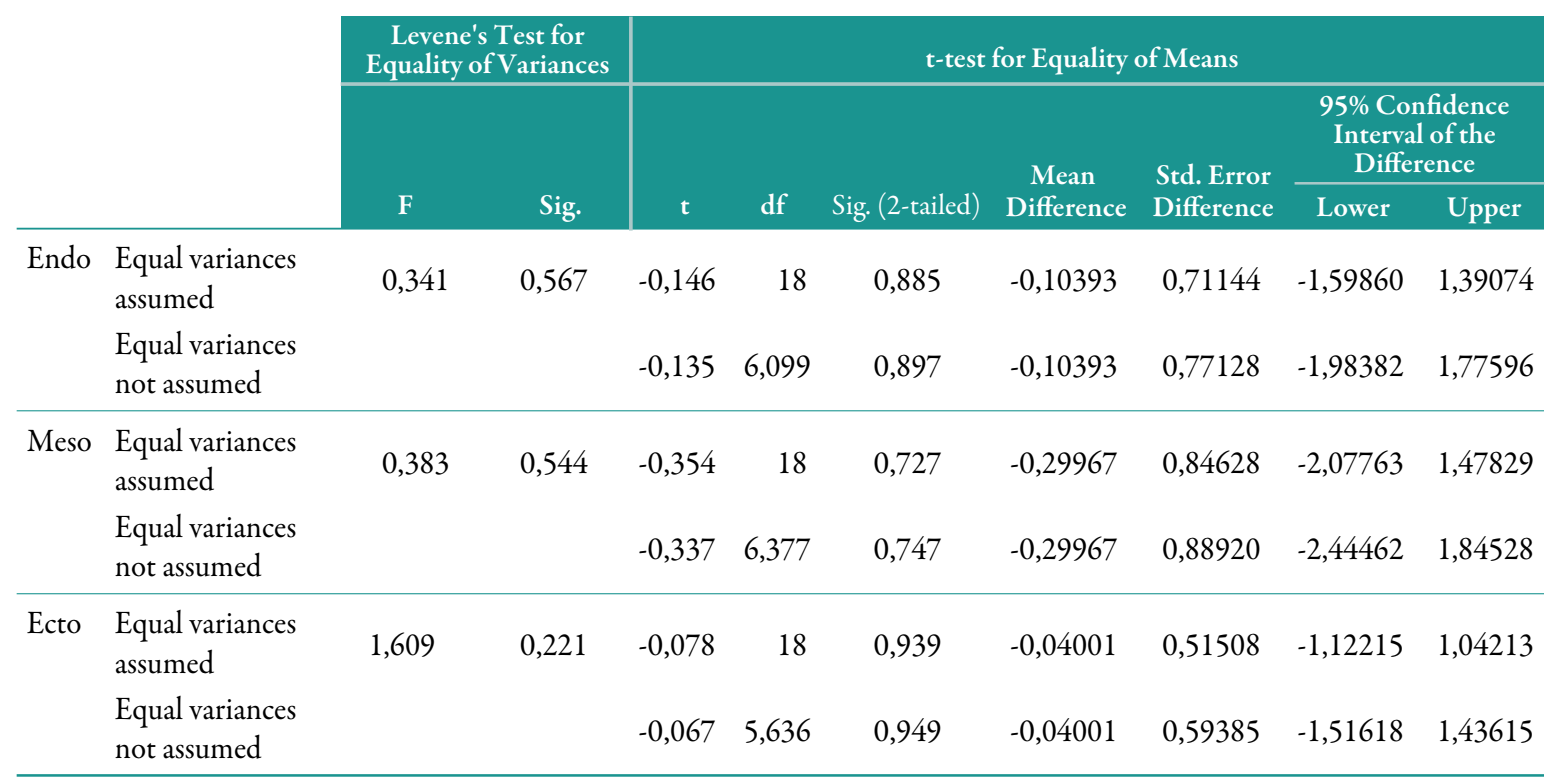


Se realizó la prueba de T de Student para comparar las poblaciones independientes de los atletas que compiten en la impulsión de bala y lanzamiento de jabalina, encontrándose que no existe una diferencia significativa en los componentes somatotípicos de ambas especialidades.

\section{Bala y Jabalina}

Independent Samples Test

\begin{tabular}{|c|c|c|c|c|c|c|c|c|c|c|}
\hline & \multicolumn{2}{|c|}{$\begin{array}{c}\text { Levene's Test for } \\
\text { Equality of Variances }\end{array}$} & \multicolumn{7}{|c|}{ t-test for Equality of Means } \\
\hline & & \multirow[b]{2}{*}{ F } & \multirow[b]{2}{*}{ Sig. } & \multirow[b]{2}{*}{$\mathbf{t}$} & \multirow[b]{2}{*}{ df } & \multirow[b]{2}{*}{ Sig. (2-tailed) } & \multirow{2}{*}{$\begin{array}{c}\text { Mean } \\
\text { Difference }\end{array}$} & \multirow{2}{*}{$\begin{array}{l}\text { Std. Error } \\
\text { Difference }\end{array}$} & \multicolumn{2}{|c|}{$\begin{array}{l}95 \% \text { Confidence } \\
\text { Interval of the } \\
\text { Difference }\end{array}$} \\
\hline & & & & & & & & & Lower & Upper \\
\hline \multirow[t]{2}{*}{ Endo } & $\begin{array}{l}\text { Equal variances } \\
\text { assumed }\end{array}$ & 0,039 & 0,847 & 0,835 & 12 & 0,420 & 0,70431 & 0,84387 & $-1,13432$ & 2,54294 \\
\hline & $\begin{array}{l}\text { Equal variances } \\
\text { not assumed }\end{array}$ & & & 0,826 & 8,147 & 0,432 & 0,70431 & 0,85234 & $-1,25503$ & 2,66366 \\
\hline \multirow[t]{2}{*}{ Meso } & $\begin{array}{l}\text { Equal variances } \\
\text { assumed }\end{array}$ & 0,269 & 0,614 & 0,120 & 12 & 0,906 & 0,11284 & 0,93840 & $-1,93176$ & 2,15744 \\
\hline & $\begin{array}{l}\text { Equal variances } \\
\text { not assumed }\end{array}$ & & & 0,118 & 7,884 & 0,909 & 0,11284 & 0,95872 & $-2,10365$ & 2,32933 \\
\hline \multirow[t]{2}{*}{ Ecto } & $\begin{array}{l}\text { Equal variances } \\
\text { assumed }\end{array}$ & 0,575 & 0,463 & $-0,692$ & 12 & 0,502 & $-0,42811$ & 0,61823 & $-1,77511$ & 0,91890 \\
\hline & $\begin{array}{l}\text { Equal variances } \\
\text { not assumed }\end{array}$ & & & $-0,662$ & 7,374 & 0,528 & $-0,42811$ & 0,64668 & $-1,94166$ & 1,08544 \\
\hline
\end{tabular}

Se realizó la prueba de T de Student para comparar las poblaciones independientes de las atletas que compiten en la impulsión de bala y martillo, encontrándose que no existe una diferencia significativa en los componentes somatotípicos de ambas especialidades

\section{Bala y Martillo}

Independent Samples Test

\begin{tabular}{|c|c|c|c|c|c|c|c|c|c|c|}
\hline & \multicolumn{2}{|c|}{$\begin{array}{l}\text { Levene's Test for } \\
\text { Equality of Variances }\end{array}$} & \multicolumn{7}{|c|}{ t-test for Equality of Means } \\
\hline & & \multirow[b]{2}{*}{ F } & \multirow[b]{2}{*}{ Sig. } & \multirow[b]{2}{*}{$\mathbf{t}$} & \multirow[b]{2}{*}{ df } & \multirow[b]{2}{*}{ Sig. (2-tailed) } & \multirow{2}{*}{$\begin{array}{c}\text { Mean } \\
\text { Difference }\end{array}$} & \multirow{2}{*}{$\begin{array}{l}\text { Std. Error } \\
\text { Difference }\end{array}$} & \multicolumn{2}{|c|}{$\begin{array}{l}\text { 95\% Confidence } \\
\text { Interval of the } \\
\text { Difference }\end{array}$} \\
\hline & & & & & & & & & Lower & Upper \\
\hline \multirow[t]{2}{*}{ Endo } & $\begin{array}{l}\text { Equal variances } \\
\text { assumed }\end{array}$ & 12.094 & 0,008 & $-1,705$ & 8 & 0,127 & $-1,23388$ & 0,72386 & $-2,90309$ & 0,43534 \\
\hline & $\begin{array}{l}\text { Equal variances } \\
\text { not assumed }\end{array}$ & & & $-1,705$ & 4,768 & 0,152 & $-1,23388$ & 0,72386 & $-3,12218$ & 0,65443 \\
\hline \multirow[t]{2}{*}{ Meso } & $\begin{array}{l}\text { Equal variances } \\
\text { assumed }\end{array}$ & 7.384 & 0,026 & $-1,885$ & 8 & 0,096 & $-1,67122$ & 0,88680 & $-3,71618$ & 0,37374 \\
\hline & $\begin{array}{l}\text { Equal variances } \\
\text { not assumed }\end{array}$ & & & $-1,885$ & 6,010 & 0,108 & $-1,67122$ & 0,88680 & $-3,84026$ & 0,49782 \\
\hline \multirow[t]{2}{*}{ Ecto } & $\begin{array}{l}\text { Equal variances } \\
\text { assumed }\end{array}$ & 52.568 & 0,000 & 1,782 & 8 & 0,113 & 0,97446 & 0,54686 & $-0,28660$ & 2,23552 \\
\hline & $\begin{array}{l}\text { Equal variances } \\
\text { not assumed }\end{array}$ & & & 1,782 & 4,095 & 0,148 & 0,97446 & 0,54686 & $-0,53001$ & 2,47893 \\
\hline
\end{tabular}


Se realizó la prueba de $\mathrm{T}$ de student para comparar las poblaciones independientes de las atletas que compiten en el lanzamiento de disco y jabalina, encontrándose que no existe una diferencia significativa entre los componentes somatotípicos de ambas especialidades

\section{Disco y Jabalina}

\begin{tabular}{|c|c|c|c|c|c|c|c|c|c|c|}
\hline \multicolumn{11}{|c|}{ Independent Samples Test } \\
\hline & & \multicolumn{2}{|c|}{$\begin{array}{c}\text { Levene's Test for } \\
\text { Equality of Variances }\end{array}$} & \multicolumn{7}{|c|}{ t-test for Equality of Means } \\
\hline & & \multirow[b]{2}{*}{ F } & \multirow[b]{2}{*}{ Sig. } & \multirow[b]{2}{*}{$\mathrm{t}$} & \multirow[b]{2}{*}{ df } & \multirow[b]{2}{*}{ Sig. (2-tailed) } & \multirow{2}{*}{$\begin{array}{c}\text { Mean } \\
\text { Difference }\end{array}$} & \multirow{2}{*}{$\begin{array}{l}\text { Std. Error } \\
\text { Difference }\end{array}$} & \multicolumn{2}{|c|}{$\begin{array}{l}95 \% \text { Confidence } \\
\text { Interval of the } \\
\text { Difference }\end{array}$} \\
\hline & & & & & & & & & Lower & Upper \\
\hline \multirow[t]{2}{*}{ Endo } & $\begin{array}{l}\text { Equal variances } \\
\text { assumed }\end{array}$ & 0,165 & 0,689 & 1,379 & 22 & 0,182 & 0,80824 & 0,58620 & $-0,40747$ & 2,02395 \\
\hline & $\begin{array}{l}\text { Equal variances } \\
\text { not assumed }\end{array}$ & & & 1,336 & 15,364 & 0,201 & 0,80824 & 0,60498 & $-0,47858$ & 2,09506 \\
\hline \multirow[t]{2}{*}{ Meso } & $\begin{array}{l}\text { Equal variances } \\
\text { assumed }\end{array}$ & 0,017 & 0,897 & 0,605 & 22 & 0,551 & 0,41251 & 0,68183 & $-1,00151$ & 1,82653 \\
\hline & $\begin{array}{l}\text { Equal variances } \\
\text { not assumed }\end{array}$ & & & 0,601 & 16,647 & 0,556 & 0,41251 & 0,68621 & $-1,03760$ & 1,86262 \\
\hline \multirow[t]{2}{*}{ Ecto } & $\begin{array}{l}\text { Equal variances } \\
\text { assumed }\end{array}$ & 0,220 & 0,644 & $-0,946$ & 22 & 0,354 & $-0,38809$ & 0,41027 & $-1,23893$ & 0,46274 \\
\hline & $\begin{array}{l}\text { Equal variances } \\
\text { not assumed }\end{array}$ & & & $-0,915$ & 15,292 & 0,374 & $-0,38809$ & 0,42402 & $-1,29037$ & 0,51419 \\
\hline
\end{tabular}

Se encontró que existe una diferencia significativa en todas las medidas de somatotipo entre las lanzadoras de disco y martillo, ya que las lan- zadoras de disco presentan menores dimensiones en sus medidas las lanzadoras de martillo.

\section{Disco y Martillo}

Independent Samples Test

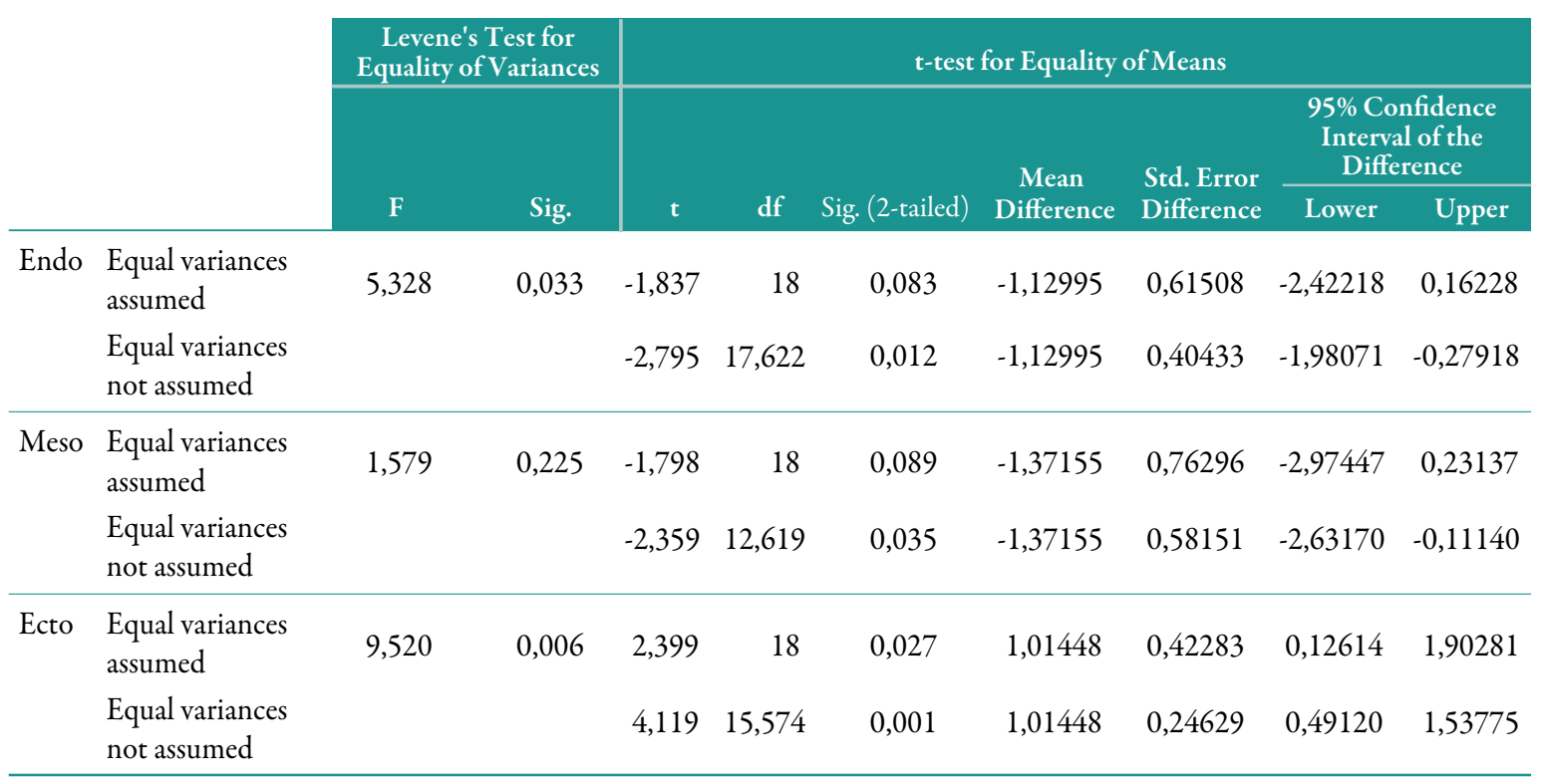


Se encontró que existe una diferencia significativa en todas las medidas de somatotipo entre las lanzadoras de jabalina y martillo, ya que las lanzadoras de jabalina presentan mayores dimensiones en sus medidas que las lanzadoras de martillo.

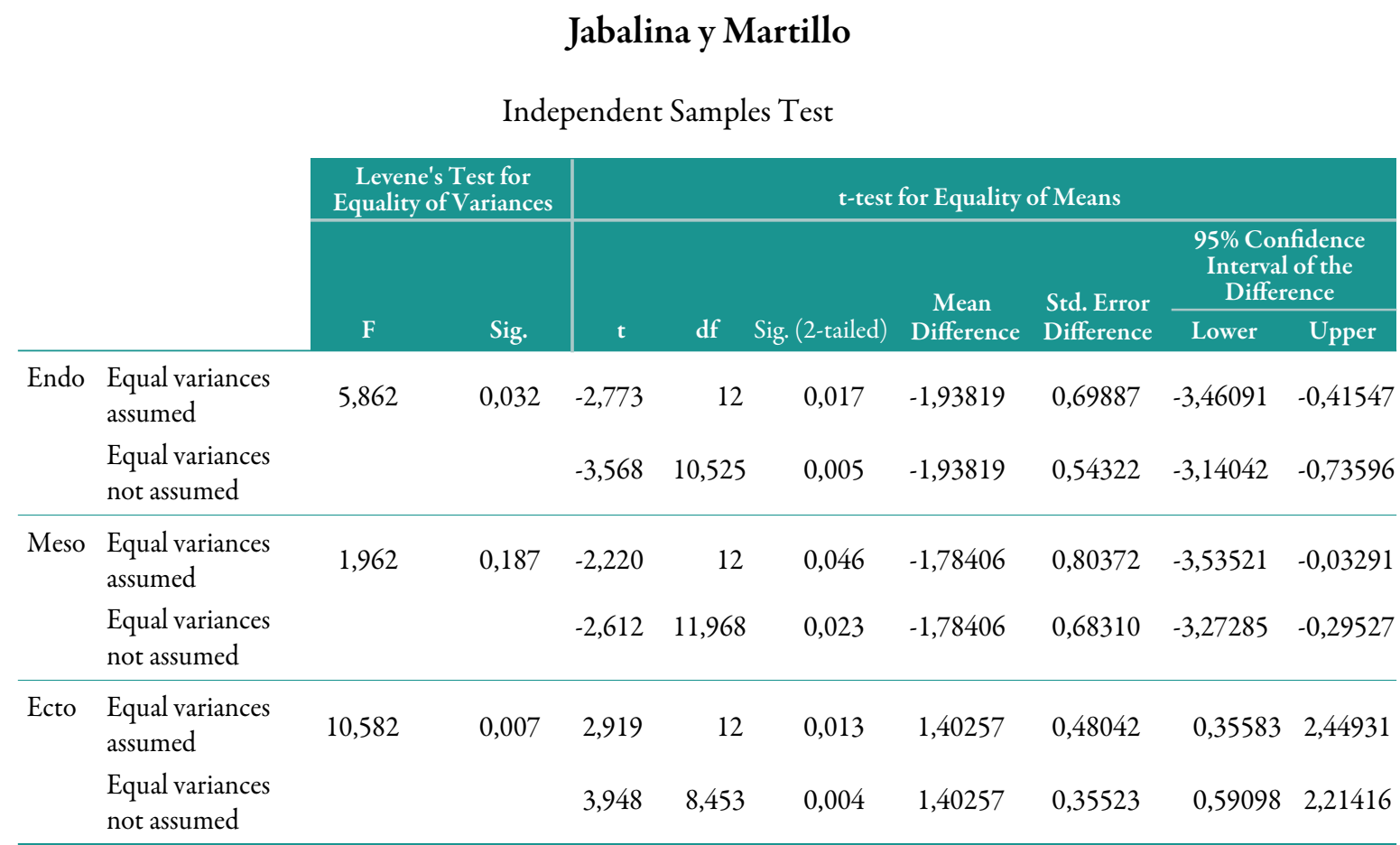

La hipótesis planteada parte de considerar modelos ideales de somatotipo obtenidos a través de la obtención de datos de deportistas de nivel mundial y olímpico. En estos modelos predomina el componente mesomórfico sobre el componente endomórfico, y por ende del ectomórfico, lo cual corrobora una serie de estudios de carácter fisiológico y biomecánico, en donde se afirma y se comprueba que la potencia, como la relación entre peso y talla, genera las acciones motrices para la impulsión de la bala.

Los datos obtenidos en el Campeonato $\mathrm{Na}$ cional 2007 describen una población totalmente ajena a esta realidad. La predominancia absoluta radica en un valor alto de la endomorfia sobre la mesomorfia y ectomorfia, considerando que el componente de endomorfia se refiere a la adiposidad relativa del atleta, es decir, representa indirectamente la masa adiposa no esencial, que no tiene otro fin fisiológico que la de asegurar una reserva energética para la supervivencia; por lo tanto, al no ser fuente de generación directa de las acciones motrices para actividades que requieren impulsión y potencia, como son las pruebas de lanzamiento, no existiría reciprocidad entre el somatotipo de las lanzadoras nacionales y sus respectivas marcas por especialidad.

Los cuadros de correlación entre los componentes del somatotipo y las marcas respectivas por especialidad dan cuenta de esta posibilidad.

\section{Resultados y comentarios de los datos recolectados}

Estadísticamente, no existe correlación entre las medidas de los componentes del somatotipo y las marcas logradas en las diferentes especialidades de lanzamiento. Sin embargo, en las lanzadoras de disco se observó una ligera correlación entre la marca alcanzada y el componente mesomórfico. En el caso de las lanzadoras de jabalina, se observó también una ligera correlación con el componente ectomórfico. En el caso de las 
atletas especialistas en lanzamiento de martillo, se encontró una diferencia significativa en sus componentes somatotípicos con las lanzadores de disco y jabalina.

\section{DISCUSIÓN Y ANÁLISIS}

Las figuras y las tablas ofrecen una comparación interesante encontrada en el estudio del somatotipo en deportistas de alto rendimiento de Argentina, realizado por Néstor A. Lentini y colaboradores.

Puede observarse que las lanzadoras femeninas de Argentina especialistas en las pruebas de lanzamiento tienen componentes promedios de endomorfia 4,7, de mesomorfia 5,3, y de ectomorfia 1,0 con una desviación de 2,3 en el componente endomórfico, 2,1 en el componente mesomórfico y 1,4 en el componente ectomórfico, dando cuenta de una característica con tendencia a la categoría mesomorfoendomórfico distinta a la de las lanzadoras nacionales que presentan una tendencia endomofo-mesomórfico. Las atletas argentinas son las que más se acercan al somatotipo ideal, teniendo también marcas superiores en ese sentido. Además, si comparamos el somatograma que relaciona el modelo de somatotipo ideal con los atletas deportistas españoles con cada somatograma por especialidad de las atletas nacionales, comprobaremos visualmente que se encuentra más cercano al área endomórfica y más lejano al área mesomórfica, y es precisa- mente en esta área donde se ubican las élites de las lanzadoras.

Podemos afirmar que la ausencia de relaciones con la intensidad propuestas en las hipótesis específicas se debe a la particular población de atletas femeninas nacionales en cuyo somatotipo predomina el factor endomórfico, lo cual representa una debilidad en el desarrollo músculoesquelético, que, aunado a la natural debilidad del desarrollo muscular femenino propio del periodo etáreo en que se encuentra, significaría que esta población de deportistas compite con una capacidad motriz y forma corporal en total desventaja, lo cual explicaría su limitada performance.

Las marcas representan el potencial de la capacidad de fuerza que genera el sistema músculo-esquelético del deportista, y si presentamos el caso de nuestras atletas nacionales que muestran una menor distribución músculo-esquelética en relación a sus longitudes corporales, frente a la mayor adiposidad relativa de estas, no es contradictorio afirmar que su performance no guarde relación con su somatotipo.

Cuando se realiza una investigación con la especialidad cineantropométrica, la finalidad es obtener información que nos dé una idea de la condición del deportista. En este caso, el análisis e interpretación de los datos nos han permitido determinar una serie de limitaciones consustanciales a la función y estructura de las lanzadoras nacionales.

\section{SOMATOTIPO MEDIOS DEPORTIVOS ARGENTINOS}

\begin{tabular}{c|c|c|c|c|c|c|c|c}
\multirow{2}{*}{ DEPORTE } & \multicolumn{4}{|c|}{ FEMENINO } & \multicolumn{4}{c}{ MASCULINO } \\
\cline { 2 - 10 } & & End. & Mess & Ect. & & End. & Mess & Ect. \\
LANZAMIENTOS & $\mathrm{X}$ & 4,7 & 5,3 & 1,0 & X & 3,4 & 5,6 & 1,2 \\
& \pm & 2,3 & 2,1 & 1,4 & \pm & 0,7 & 1,7 & 1,0 \\
\hline
\end{tabular}




\section{CONCLUSIONES}

1. La relación del somatotipo femenino en relación con las marcas obtenidas en las pruebas de lanzamiento es mínima.

2. El $60 \%$ de lanzadoras de disco presentan un somatotipo donde predomina el componente endomórfico.

3. El $58 \%$ de lanzadoras de bala presentan un somatotipo donde predomina el componente endomórfico.

4. En la prueba de jabalina, el $45 \%$ de lanzadoras presenta un somatotipo donde predomina el componente endomórfico.

5. En la prueba de martillo, el $80 \%$ de lanzadoras presenta el somatotipo donde predomina el componente endomórfico.

6. En todas las pruebas de lanzamiento de disco, jabalina, martillo e impulsión de bala, las atletas presentaron una predominancia del componente endomórfico.

7. Las pruebas de lanzamiento están determinadas por la capacidad de fuerza, potencia, elasticidad, velocidad y potencia del sistema músculo-esquelético, la cual se ve disminuida en la forma corporal del atleta nacional. Esto revelaría la ausencia de reciprocidad entre el somatotipo y las marcas de las atletas, en las especialidades correspondientes.

8. El atleta nacional presenta el componente predominante de endomorfia y el somatotipo ideal es mesomórfico.

9. Este trabajo contiene datos antropométricos de referencia, tanto normativos como de prototipo, es decir, es nuestra realidad, y en este caso es específico, ya que se trata de las lanzadoras del Perú. Son parte de una herramienta de mucha utilidad para el profesional del deporte y de la actividad física. Además, debe considerarse siempre la importancia de seguir normas metrológicas en su generación, como las elaboradas por la Sociedad Internacional para Avances en Cineatropometría (ISAK).
10. En nuestra muestra estaban incluidas atletas mujeres solteras y casadas, y no hay diferencias entre ellas.

11. Se ha demostrado que en el somatotipo de las atletas lanzadoras mujeres en el Perú tiene predominio el componente endomórfico. Este discernimiento es un dato muy importante para entender el bajo rendimiento a nivel sudamericano y mundial en esta prueba.

12. Hemos demostrado que las pruebas de lanzamiento dependen de la fuerza, de la potencia, de la velocidad, del sistema músculo-esquelético, y que están disminuidas de la forma corporal de las atletas de las diferentes regiones del país.

13. La información científica que genera la cineantropometría para el deporte busca establecer modelos ideales de forma, composición y proporcionalidad corporal en relación a la performance de las diversas especialidades.

14. En este caso, se ha revelado que el predominio endomórfico en el somatotipo de las lanzadoras nacionales estaría afectando directamente sus marcas.

15. Lo anterior no desdice lo establecido a nivel internacional acerca de que el somatotipo ideal del lanzador está basado predominantemente en el componente mesomórfico.

16. Esto nos demuestra que en el caso de las pruebas de lanzamiento en el deporte del atletismo, las lanzadoras actuales no conseguirán mejoras sustanciales en los diferentes eventos en los cuales el país participe.

17. Se ha podido observar que las atletas mayores no llegan con un buen rendimiento y son superadas por las juveniles en rendimiento y marcas.

18. Nuestro aporte también consiste en haber determinado que el más alto porcentaje de deportistas en esta especialidad se encuentra en la costa, con diferencias significativas en- 
tre sierra y selva, teniendo en cuenta todas las clasificaciones de las regiones.

19. Descubrimos también que la vocación de la especialidad de martillo se da en el $80 \%$ de las deportistas.

\section{RECOMENDACIONES}

1. Se debe detectar una nueva generación de deportistas que reúnan los requisitos previos de condición física y forma corporal, con la finalidad de formarlos para que se acerquen a la performance internacional y compitan en mejores condiciones.

2. Es preciso mejorar el nivel de conocimientos de los entrenadores, capacitándolos con cursos sobre entrenamiento deportivo específico para lanzadores de disco, martillo jabalina e impulsión de la bala.

3. Capacitar a los entrenadores en un curso sobre cineantropometría, a fin de que sus conocimientos puedan ser utilizados en bien de los alumnos y para mejorar los resultados de los lanzamientos en el Perú.

4. Se recomienda utilizar técnicas para desarrollar la fuerza, la potencia, la flexibilidad y la velocidad del sistema músculo-esquelético del atleta nacional.

5. Planificar jornadas de educación física y deportes y reflexionar sobre los sistemas de entrenamiento y aplicación de las ciencias del deporte, así como promover resultados y marcas de las lanzadoras a nivel nacional e internacional.

6. Nuestra fortaleza está en el lanzamiento de martillo (80\%) y disco (60\%), participantes que son indicadores importantes para promover estos lanzamientos en las diferentes regiones del Perú.

7. Adecuar los campos para las pruebas de lanzamiento en los parques zonales e instituciones educativas, con la finalidad de difundir el aprendizaje de estas pruebas bajo la dirección de entrenadores expertos en la materia.

8. Implementar un laboratorio cineantropométrico en la Escuela Profesional de Ciencias del Deporte de la Universidad Alas Peruanas, para realizar investigaciones en los diferentes deportes y brindar servicio a las diferentes instituciones deportivas que así lo requieran.

\section{REFERENCIAS BIBLIOGRÁFICAS}

1. Abadía Colas, Francisco; Adell Castan, José Antonio; Amador Ramírez. Diccionario Paidotribo de la Actividad Física y el Deporte, Vol. I A y B. Editorial Paidotribo, 1ra Edición. Edición 1999.

2. Asociación Internacional de Federaciones de Atletismo - I.A.A.F. Manual 2002 2003. Editotrial: Mónaco Cedex 17 rue Princesse Florestine Manual. España 2002 - 2003 Oficial.

3. Alexander, Pedro (1995). Aptitud Física, Caracteristicas Morfológicas y Composición Corporal. Editorial Depoaction, Venezuela.

4. Ballesteros J.M.; Álvarez J. Manual Didáctico del Atletismo. Editorial Real Federación Española de Atletismo, año 1992.

5. Bernhard, Günter (1983). El Entrenamiento del Atleta Juvenil. Edit. Montigraf, Lima, Perú.

6. Congreso E.A.C.A. (1995). Cuadernos de Atletismo $N^{\circ} 35$. Pruebas Combinadas. Real Federación Española de Atletismo Escuela Nacional de...

7. Entrenadores Centro de Real Federación Española de Atletismo. Escuela Nacional de Entrenadores. Centro de Documentación, 1995.

8. Federación Peruana de Atletismo. Memorias Sudamericano de Atletismo._Editorial Brasa S.A. Año 1995.

9. George, James y otros (1996). Tests y Pruebas Fisicas. 1ra. Edición, Edit. Paidotribo, España. 
10. Geoffrey Dyson. Mecánica del Atletismo. Ministerio de Cultura, Consejo Superior de Deportes

11. Gil Francisco; Pascua Manuel; Sánchez Rafael. Manual Básico de Atletismo._Editado por la Real Federación Española de Atletismo, Impreso por Industrias Gráficas Omnia. Año 2000, España.

12. Herbert Hopf; Martín Hillebrecht; Natascha Schmidt; Peter Thompson. Técnicas del Atletismo y progresión de enseñanza. Sistema de formación y certificación de entrenadores (IAAF).

13. Hernández Corvo, Roberto (1987). Morfologia Funcional Deportiva. Edit. CientíficoTécnica, Cuba.

14. Instituto Peruano del Deporte. Reglamento Internacional de Atletismo. 1ra Edición. Editado por I.A.A.F., 1984.

15. J. L. Thompson M.SC., Peter. Introducción a la Teoría del Entrenamiento. Federación Internacional de Atletismo Amateur (I.A.A.F.). Impreso por Marhallarts Print services L.T.D. Año 1991.

16. James D. George; A. Garth Fisher; Pat R. Vehrs. Tesis pruebas fisicas. Editorial Paidotribo, 1996. 1ra Edición, España.

17. J. Duncan Mac Dowall; Howard A.W. y otros. Evaluación Fisiológica del Deportista. Paidotribo, Fitnees, 1980.

18. Jack H. Wilmore; David L. Costill. Fisiologia del Esfuerzo y del Deporte._Editorial Paidotribo, 1984-1990.
19. López Chicharro; Almudena Fernández V. Fisiología del Ejercicio. Editorial Panamerica, 2001.

20. Luque Marco A.; Willi Gerriemann. Manual del Entrenamiento Atlético.

21. Martínez José Luis. Disco, Martillo y Pruebas Combinadas. Grefol S.A., Madrid, 1982. Federación Española de Atletismo. Escuela Nacional de entrenadores.

22. Mashiro Suganuma; Eiji Ikehara. Estudio de la Morfología y la Aptitud Fisica. Editorial Cooperación Técnica Perú- Japón, 1988. Editorial Convenio Boliviano -Alemán, 2002.

23. Matvéiev, L. El proceso del entrenamiento deportivo._Editorial Stadium, 1983. Argentina.

24. Platanov, Vladimir (1993). El Entrenamiento Deportivo: Teoría y Metodología. Barcelona, España, Editorial Paidotribo.

25. Rius Sant, Joan. Metodología del atletismo. Editorial Paidotribo. Segunda edición, Barcelona, 1993.

26. Rodríguez Velásquez, Jaime Ricardo. Diccionario Enciclopédico y Técnicas Deportivas. Editorial Centro de Emprendimiento Empresarial, 1995.

27. Rodríguez Velásquez, Jaime Ricardo. El Entrenamiento Deportivo en el Perú.3ra Edición, año 1992.

28. Salazar, Teodoro. Atletismo Peruano. Editorial Salesiana, Lima,Perú, 1990.

29. Verjoshanski, Lurig (1990). Entrenamiento Deportivo. Barcelona, España, Editorial Martínez Roca, S.A. 


\section{FUENTES DE INTERNET}

1. Erick A. Salas Ramírez. Caracteristicas Antropométricas en Seleccionadas de Vóleibol Femenino de Perú Categoría Menores. Artículo Pid: 731, 06/11/2006 bttp://www.sobreentrenamiento.com/ PubliCE/Articulo.asp?ida=731 \&t $p=s$

2. NéstorA.Lentini. EstudioSomatotipicoenDeportistasde AltoRendimientodeArgentina. 27/11/2006. Pid: 738 bttp://www.sobreentrenamiento.com/PubliCE/Articulo.asp?ida=738\&t $p=s$

3. Robert M. Malina Antropometría. 16/10/2006. Pid: 718. bttp://www.sobreentrenamiento.com/PubliCE/Articulo.asp?ida=718\&tp=s

4. Carter, Lindsay J. Factores Morfológicos que limitan el Rendimiento Humano. PubliCE Standard. 28/07/2003. Pid: 139.

bttp://www.sobreentrenamiento.com/PubliCE/Articulo.asp?ida=139ひtp=s

5. Parajón Víscido, Manuel. La Evaluación Antropométrica. PubliCE Standard. 22/11/2002. Pid: 21. bttp://www.sobreentrenamiento.com/PubliCE/Articulo.asp?ida=21 \&t $p=s$

6. Brito, Pedro. Somatotipo Antropométrico Heath-Carter bttp://www.rendeportin.com.ve/portal/modules.php?name $=$ Newséfile=article\& $s i d=2$ 